\title{
STABLE TRACE FORMULA: CUSPIDAL TEMPERED TERMS
}

\author{
ROBERT E. KOTTWITZ
}

Consider a connected reductive group $G$ over a number field $F$. For technical reasons we assume that the derived group of $G$ is simply connected (see [L1]). In [L3] Langlands partially stabilizes the trace formula for $G$. After making certain assumptions, he writes the elliptic regular part of the trace formula for $G$ as a linear combination of the elliptic $G$-regular parts of the stable trace formulas for the elliptic endoscopic groups $H$ of $G$. The function $f^{H}$ used in the stable trace formula for $H$ is obtained from the function $f$ used in the trace formula for $G$ by transferring orbital integrals.

Langlands uses $\iota(G, H)$ to denote the coefficient of the stable trace formula of $H$ in the linear combination referred to above. He obtains an explicit formula for $\iota(G, H)$, which we review in Section 8 .

Eventually it should be possible to stabilize the whole trace formula for $G$. In Section 12 we sketch the stabilization of the cuspidal tempered part of the trace formula for $G$. To carry this out we are forced to make a number of assumptions, some of which are unlikely to be verified in the near future, but this is not as serious as it appears at first, for our only purpose in Section 12 is to increase our understanding of the formalism that underlies the stable trace formula. In any case, we are able to prove a small part of what we need, and we put these results in Section 11.

Although the stabilization carried out in Section 12 is speculative, it leads us to a new expression for $\iota(G, H)$. In Section 8 we prove that this expression agrees with the one found by Langlands. This agreement is encouraging and should, perhaps, be regarded as evidence in favor of the assumptions in Section 12. Our expression for $\iota(G, H)$ is

$$
\iota(G, H)=\tau_{1}(G) \cdot \tau_{1}(H)^{-1} \cdot \lambda^{-1} .
$$

The number $\lambda$ also appears in Langlands's expression for $\iota(G, H)$; its definition can be found in 8.1 . The number $\tau_{1}(G)$ is given by

$$
\left|\pi_{0}\left(Z(\hat{G})^{\Gamma}\right)\right| \cdot\left|\operatorname{ker}^{1}(F, Z(\hat{G}))\right|^{-1}
$$

We need to explain the symbols used in this formula. We write $\hat{G}$ instead of ${ }^{L} G^{0}$,

Received June 24, 1983. Partially supported by the National Science Foundation under Grant MCS 82-00785. 
and we write $Z(\hat{G})$ for the center of $\hat{G}$. The superscript $\Gamma$ stands for invariants under $\Gamma$, the absolute Galois group of $F$, and $\pi_{0}\left(Z(\hat{G})^{\Gamma}\right)$ stands for the group of connected components of $Z(\hat{G})^{\Gamma}$. The vertical bars are used to denote cardinality of a finite set. Finally, $\operatorname{ker}^{1}(F, Z(\hat{G}))$ is the kernel of

$$
H^{1}(F, Z(\hat{G})) \rightarrow \prod_{v} H^{1}\left(F_{v}, Z(\hat{G})\right),
$$

where the product is taken over all places $v$ of $F$. A remark of Shelstad, mentioned on p. 169 of [L3], leads us to expect a connection with Tamagawa numbers. In fact, $\tau_{1}(G)$ turns out to be the relative Tamagawa number of $G$ (the quotient of the Tamagawa number of $G$ by the Tamagawa number of the simply connected cover of the derived group of $G$ ), as we prove in Section 5. Our formula generalizes formulas of Ono [O] for tori and semisimple groups and is related to a formula of Sansuc [Sa].

This formula for the relative Tamagawa number is a special case of the following general principle. Consider an invariant of connected reductive groups over a local or global field. Assume that the invariant is trivial for semisimple simply connected groups. Then it should be possible to compute the invariant of $G$ from the Galois module $Z(\hat{G})$. In this paper we will consider two more illustrations of this principle. In Section 4 we consider a number field $F$ and the invariant $\operatorname{ker}^{1}(F, G)$, the kernel of the Hasse map

$$
H^{1}(F, G) \rightarrow \prod_{v} H^{1}\left(F_{v}, G\right)
$$

For groups with no $E_{8}$ factors we show that there is a canonical bijection from $\operatorname{ker}^{1}(F, G)$ to the dual of the finite abelian group $\operatorname{ker}^{1}(F, Z(\hat{G}))$. In Section 6 we consider a $p$-adic field $F$ and the invariant $H^{1}(F, G)$. We show that there is a canonical bijection from $H^{1}(F, G)$ to the dual of the finite abelian group $\pi_{0}\left(Z(\hat{G})^{\Gamma}\right)$.

These results run parallel to results of Sansuc [Sa], who uses $\mathrm{Pic} G$ and $\mathrm{Br}_{a} G$ (a certain subquotient of $\operatorname{Br} G)$, rather than $Z(\hat{G})$. In Section 2 we relate the two points of view by showing that $\operatorname{Pic} G=\pi_{0}\left(Z(\hat{G})^{\Gamma}\right)$ and $\operatorname{Br}_{a} G=H^{1}(F, Z(\hat{G}))$ for any connected reductive group $G$ over an arbitrary field $F$. Sansuc also studies the failure of weak approximation. It is easy to reformulate his results in terms of $Z(\hat{G})$; we leave this to the reader.

The proof of the agreement of the two expressions for $\iota(G, H)$ is an exercise in Tate-Nakayama duality [T1], [T2]. For the convenience of the reader, we review this theory in Section 3, in the form best suited to our applications.

In Sections 1, 7, 10 we review $L$-groups, endoscopic groups and admissible homomorphisms. It is necessary to have a firm grasp on these before attempting the stabilization in Section 12. We have made what we hope are some technical improvements in the presentation of this material-for example, our endoscopic data are pairs, rather than sextuples, and our $L$-groups are not rigidified by 
choosing splittings. One effect of these modifications is that it is easier to handle isomorphisms and automorphisms, which works to our advantage in Section 11.

Section 9 gives a new approach to the main construction of Ch. VII of [L3]. The problem and its solution are stated in 9.2 and 9.3. In 9.5 we give a new approach to something else in Ch. VII of [L3]: a global obstruction to transferring a maximal $F$-torus in a quasi-split group $H$ to an inner form $G$.

Three final comments are needed. First, it should be noted that algebraists will find little that is new in this paper, since our results on $\operatorname{ker}^{1}(F, G)$ and Tamagawa numbers are simply restatements of Sansuc's results, using $Z(\hat{G})$ rather than Pic $G$ and $\operatorname{Br}_{a} G$. Those interested in the trace formula, however, may find our restatements useful, and it is for these readers that the remarks in 4.4, 5.3 are intended. As the remarks suggest, this paper has been written so as to give the reader a choice between translating Sansuc's results (this is shorter) and reproving them, by parallel methods, in terms of $Z(\hat{G})$ rather than Pic $G$ and $\mathrm{Br}_{a} G$ (this is longer, but may seem more natural to those interested in the trace formula). In any case, the proofs require all of the important theorems on the Galois cohomology of algebraic groups over local and global fields, and in particular they give no new insight into the Hasse principle.

Second, given the speculative nature of Section 12, it may be worthwhile to explain its origin. I wanted to see how Langlands's numbers $\iota(G, H)$ would show up in the cuspidal tempered part of the trace formula, so I made some plausible assumptions about the form of this part of the trace formula and then stabilized it. This led to the new expression for $\iota(G, H)$ given above, and the new expression turned out to be correct. In proving this I was led to express $\operatorname{ker}^{1}(F, G)$ and $\tau_{1}(G)$ in terms of $Z(\hat{G})$. After finding out about similar results of Sansuc, I realized that it should also be possible to express $\operatorname{Pic} G$ and $\operatorname{Br}_{a} G$ in terms of $Z(\hat{G})$, and it turned out to be easy to check this. But in writing this paper I have reversed the whole procedure, so that $\operatorname{Pic} G, \mathrm{Br}_{a} G$ come first and the stabilization comes last.

Third, I would like to thank the referee for pointing out that the general definition of $\operatorname{Ext}_{\Gamma}^{n}(A, B)$ in 2.1 needed to be reformulated.

Notation and conventions. We use $F$ to denote a field (often a number field). Let $\bar{F}$ be an algebraic closure of $F$ and write $F_{\text {sep }}$ for the separable closure of $F$ in $\bar{F}$. We denote by $\Gamma$ the Galois group of $F_{\text {sep }}$ over $F$. If $F$ is a number field, we write $A_{\bar{F}}$ for the direct limit of the adele rings $A_{L}$, where $L$ runs over the finite extensions of $F$ contained in $\bar{F}$.

We consider only discrete $\Gamma$-modules $A$. This means that $\operatorname{Stab}_{\Gamma}(a)$ is open in $\Gamma$ for all $a \in A$.

By an induced $\Gamma$-module we mean a $\Gamma$-module that has a finite $\Gamma$-stable Z-basis. We say that an $F$-torus $T$ is induced if $X^{*}(T)$ is an induced $\Gamma$-module. Let $S$ be a $\mathrm{C}$-torus on which $\Gamma$ acts. We say that $S$ is induced if the $\Gamma$-module $X^{*}(S)$ is induced. 
Let $S$ be a set on which $\Gamma$ acts. We write $S^{\Gamma}$ for $\{s \in S: \sigma \cdot s=s$ for all $\sigma \in \Gamma$. Let $A$ be a $\Gamma$-module. We write $H^{i}(F, A)$ instead of $H^{i}(\Gamma, A)$.

Let $G$ be a group. We denote by $Z(G)$ the center of $G$ and by $\operatorname{Out}(G)$ the group of outer automorphisms of $G$. For $g \in G$ we let $\operatorname{Int}(g)$ denote the inner automorphism $x \mapsto g x g^{-1}$ of $G$.

We write $A^{D}$ for the character group of a locally compact abelian group $A$. Sometimes our characters have values in $Q / Z$, in which case we use the exponential mapping $x \mapsto \exp (2 \pi i x)$ from $\mathrm{Q} / \mathrm{Z}$ to $\mathrm{C}^{\times}$to view them as complex-valued characters.

Let $G$ be a connected reductive group over $F$. For a field extension $K$ of $F$ we write $G_{K}$ for the $K$-group obtained from $G$ by extension of scalars. Let $G_{\text {der }}$ denote the derived group $G$, let $G_{\text {sc }}$ denote the simply connected cover of $G_{\mathrm{der}}$, and let $G_{\text {ad }}$ denote the adjoint group of $G$. Let $T$ be a maximal torus of $G$. We will write $T_{\mathrm{der}}$ for the intersection of $T$ with $G_{\mathrm{der}}, T_{\mathrm{sc}}$ for the inverse image of $T_{\mathrm{der}}$ in $G_{\text {sc }}$, and $T_{\text {ad }}$ for the image of $T$ in $G_{\text {ad }}$. We write $\hat{G}$ for the connected Langlands dual group of $G$, rather than ${ }^{L} G^{0}$. We need the notion of a $z$-extension; for this we refer the reader to $[\mathrm{K}]$.

We write $\pi_{0}(X)$ for the set of connected components of a topological space $X$. We use this only for topological groups $G$, in which case $\pi_{0}(G)$ is the group $G / G^{0}$, where $G^{0}$ is the identity component of $G$.

We write $|X|$ for the cardinality of a finite set $X$.

Let $X$ be an algebraic variety over $F$. Following [Sa], we define $\operatorname{Br}_{a} X$ to be the following subquotient of the Brauer group $\operatorname{Br} X$ of $X$. Let $\operatorname{Br}_{1} X$ denote the kernel of $\operatorname{Br} X \rightarrow \operatorname{Br}\left(X_{F_{\text {sep }}}\right)$, and let $\mathrm{Br}_{0} X$ denote the image of $\operatorname{Br} F \rightarrow \operatorname{Br} X$. Then we put $\mathrm{Br}_{a} X$ equal to the quotient $\mathrm{Br}_{1} X / \mathrm{Br}_{0} X$. For an algebraic group $G$ over $F$ we have the rational point $e \in G(F)$, and from the corresponding morphism $\operatorname{Spec} F \rightarrow G$ we get a homomorphism $\operatorname{Br} G \rightarrow \operatorname{Br} F$. We write $\operatorname{Br}_{e} G$ for the intersection of $\mathrm{Br}_{1} G$ with the kernel of $\mathrm{Br} G \rightarrow \mathrm{Br} F$. The obvious homomorphism $\mathrm{Br}_{e} G \rightarrow \mathrm{Br}_{a} G$ is an isomorphism.

1. Review of $L$-groups. In this paper we will not rigidify our $L$-groups by choosing splittings. To avoid confusion later we need to state our conventions regarding $L$-groups. Our presentation is based on Borel's [B].

1.1. Let $G$ be a connected reductive group over an algebraically closed field. Consider pairs $(T, B)$ where $T$ is a maximal torus of $G$ and $B$ is a Borel subgroup of $G$ that contains $T$. Given two pairs $\left(T_{1}, B_{1}\right)$ and $\left(T_{2}, B_{2}\right)$, the inner automorphisms $\alpha$ of $G$ such that $\alpha\left(T_{1}\right)=T_{2}, \alpha\left(B_{1}\right)=B_{2}$ all induce the same isomorphism $T_{1} \stackrel{\sim}{\rightarrow} T_{2}$. In particular, we have canonical isomorphisms $X^{*}\left(T_{1}\right)$ $\tilde{\rightarrow} X^{*}\left(T_{2}\right)$ and $X_{*}\left(T_{1}\right) \stackrel{\sim}{\rightarrow} X_{*}\left(T_{2}\right)$. Let $X^{*}$ (resp. $X_{*}$ ) denote the projective limit of the groups $X^{*}(T)$ (resp. $X_{*}(T)$ ), where the limit is taken over the set of pairs $(T, B)$. Let $\Delta^{*}$ (resp. $\Delta_{*}$ ) denote the projective limit of the sets $\Delta^{*}(T, B)$ (resp. $\left.\Delta_{*}(T, B)\right)$, where $\Delta^{*}(T, B)$ is the set of simple $B$-positive roots of $T$, and $\Delta_{*}(T, B)$ is the set of simple $B$-positive coroots of $T$. Then $\left(X^{*}, \Delta^{*}, X_{*}, \Delta_{*}\right)$ is a based root datum, which we will denote by $\Psi_{0}(G)$. 
1.2. Let $G$ be a connected reductive group over $F$. Then $\Gamma$ acts on $\Psi_{0}\left(G_{\bar{F}}\right)$. We will denote by $\Psi_{0}(G)$ the based root datum $\Psi_{0}\left(G_{\bar{F}}\right)$ together with the action of $\Gamma$.

1.3. A splitting of a connected reductive group $G$ is a triple $(T, B$, $\left\{X_{\alpha}\right\}_{\alpha \in \Delta^{*}(T, B)}$ ), where $T$ is a maximal torus of $G, B$ is a Borel subgroup of $G$ that contains $T$, and $X_{\alpha}$ is a nonzero element of the root space $\operatorname{Lie}(G)_{\alpha}$. The group $\operatorname{Aut}(G)$ acts on the set of splittings; the subgroup $G_{\text {ad }}$ of $\operatorname{Aut}(G)$ acts simply transitively.

1.4. Let $H$ be a connected reductive group over $\mathrm{C}$ on which $\Gamma$ acts. Then $\Gamma$ acts on $\Psi_{0}(H)$. If $\Gamma$ fixes some splitting of $H$, then we will say that the action is an L-action.

1.5. Let $G$ be a connected reductive group over $F$. A dual group for $G$ is a connected reductive group $\hat{G}$ over $C$ together with an $L$-action of $\Gamma$ on $\hat{G}$ and a $\Gamma$-isomorphism from $\Psi_{0}(\hat{G})$ to the dual of $\Psi_{0}(G)$. The group $\hat{G}$ is the identity component of the $L$-group ${ }^{L} G$ and in Borel [B] is denoted by ${ }^{L} G^{0}$. Since we have not chosen a splitting of $\hat{G}$, our version of the dual group is not rigid. Its automorphism group is $\left[(\hat{G})_{\mathrm{ad}}\right]^{\Gamma}$. It follows from the next lemma that these automorphisms are harmless.

1.6. LEMMA. The canonical homomorphism $\hat{G}^{\Gamma} \rightarrow\left[(\hat{G})_{\mathrm{ad}}\right]^{\Gamma}$ is surjective.

Let $g$ be an element of $\hat{G}$ whose image in $(\hat{G})_{\text {ad }}$ is fixed by $\Gamma$. For $\sigma \in \Gamma$ there exists $z_{\sigma} \in Z(\hat{G})$ such that $g^{\sigma}=z_{\sigma} g$. Choose a splitting $\left(\hat{T}, \hat{B},\left\{X_{\alpha}\right\}\right)$ that is fixed by $\Gamma$. The double coset of $\hat{B}$ in $\hat{G}$ containing $g$ is fixed by $\Gamma$. Using Lemma 6.2 of [B], we see that there exists $w \in \hat{G}^{\Gamma}$ such that $w$ normalizes $\hat{T}$ and $g \in \hat{B} w \hat{B}$. Let $\hat{N}$ denote the unipotent radical of $\hat{B}$. Then $g$ has a unique Bruhat decomposition $g=u w v t$ where $u \in \hat{N} \cap{ }^{w} \hat{N}^{-}, v \in \hat{N}, t \in \hat{T}$. But $g=g^{\sigma} z_{\sigma}^{-1}=u^{\sigma} w v^{\sigma}\left(t^{\sigma} z_{\sigma}^{-1}\right)$, and since $\sigma$ preserves $\hat{T}, \hat{N}$ and $w$, we see that $u, v \in \hat{G}^{\Gamma}$ and $t^{\sigma}=z_{\sigma} t$. Thus $g$ can be written as $g=h t$ with $h \in \hat{G}^{\Gamma}$ and $t \in \hat{T}$. Let $\hat{S}$ denote the image of $\hat{T}$ in $(\hat{G})_{\text {ad }}$. The image of $t$ in $\hat{S}$ lies in $\hat{S}^{\Gamma}$. But $X^{*}(\hat{S})$ has a basis preserved by $\Gamma$, namely the set of simple $\hat{B}$-positive roots of $\hat{S}$, and therefore $\hat{S}^{\Gamma}$ is connected. From this it is clear that $\hat{T}^{\Gamma} \rightarrow \hat{S}^{\Gamma}$ is surjective, which implies that there exists $z \in Z(\hat{G})$ such that $t z \in \hat{T}^{\Gamma}$. We see immediately that $g z \in \hat{G}^{\Gamma}$.

1.7. Corollary. Any two splittings of $\hat{G}$ that are fixed by $\Gamma$ are in the same orbit under $\hat{G}^{\Gamma}$.

Let $y_{1}, y_{2}$ be the two splittings, and let $\alpha$ be the unique inner automorphism of $\hat{G}$ such that $\alpha \cdot y_{1}=y_{2}$. Then $\alpha^{\sigma} \cdot y_{1}=y_{2}$ for all $\sigma \in \Gamma$, since $y_{1}, y_{2}$ are fixed by $\Gamma$. Therefore $\alpha \in\left[\hat{G}_{\mathrm{ad}}\right]^{\Gamma}$, and the lemma implies that there exists $g \in \hat{G}^{\Gamma}$ such that $\operatorname{Int}(g)=\alpha$.

1.8. Let $G, H$ be connected reductive groups over $F$. We will say that an $F$-homomorphism $\alpha: G \rightarrow H$ is normal if $\alpha(G)$ is normal in $H$ and $G \rightarrow \alpha(G)$ is separable. Choose splittings of $\hat{G}$ and $\hat{H}$. Then there is a unique $\Gamma$ homomorphism $\hat{\alpha}: \hat{H} \rightarrow \hat{G}$ compatible with the splittings and with the map $\Psi_{0}(\hat{H}) \rightarrow \Psi_{0}(\hat{G})$ dual to $\Psi_{0}(G) \rightarrow \Psi_{0}(H)$. Changing the choice of splittings (see 
1.7) replaces $\hat{\alpha}$ by $\operatorname{Int}(g) \circ \hat{\alpha}$ for some $g \in \hat{G}^{\Gamma}$. This means that the $\hat{G}^{\Gamma}$-conjugacy class of $\hat{\alpha}: \hat{H} \rightarrow \hat{G}$ is canonical. In particular, the homomorphism $Z(\hat{H}) \rightarrow Z(\hat{G})$ induced by $\hat{\alpha}$ is canonical. Thus we get a contravariant functor $G \mapsto Z(\hat{G})$ from the category of connected reductive $F$-groups and normal $F$-homomorphisms to the category of diagonalizable $\mathrm{C}$-groups with $\Gamma$-action. Furthermore, if $1 \rightarrow G_{1} \rightarrow G_{2} \rightarrow G_{3} \rightarrow 1$ is exact, then so is

$$
1 \rightarrow Z\left(\hat{G}_{3}\right) \rightarrow Z\left(\hat{G}_{2}\right) \rightarrow Z\left(\hat{G}_{1}\right) \rightarrow 1 .
$$

The following facts are also useful.

(1.8.3) The derived group of $G$ is simply connected if and only if $Z(\hat{G})$ is connected, in which case $Z(\hat{G})=\hat{D}$, where $D=G / G_{\mathrm{der}}$.

(1.8.4) Suppose that $G$ is semisimple, and let $C$ be the kernel of $G_{\text {sc }} \rightarrow G$. Then $Z(\hat{G})=X^{*}(C)$.

2. Ext, Pic and Br. In this section $F$ is an arbitrary field and $\Gamma$ $=\operatorname{Gal}\left(F_{\text {sep }} / F\right)$. In $2.1,2.2,2.3$ the group $\Gamma$ could just as well be an arbitrary profinite group.

2.1. Let $A, B$ be $\Gamma$-modules. We define $\operatorname{Ext}_{\Gamma}^{n}(A, B)$ to be the direct limit of the groups $\operatorname{Ext}_{\mathrm{Z}[\Gamma / N]}^{n}\left(A_{N}, B^{N}\right)$, where $N$ runs over the set of open normal subgroups of $\Gamma\left(A_{N}\right.$ denotes the coinvariants of $N$ on $\left.A\right)$.

2.2. LemMA. Let $M$ be a $\Gamma$-module that is finitely generated as an abelian group. Let $D$ be the diagonalizable $\mathrm{C}$-group $\operatorname{Hom}_{\mathrm{Z}}\left(M, \mathrm{C}^{\times}\right)$. Then $X^{*}(D)=M$ and

$$
\operatorname{Ext}_{\Gamma}^{n}(M, \mathrm{Z})= \begin{cases}X_{*}(D)^{\Gamma} & n=0 \\ \pi_{0}\left(D^{\Gamma}\right) & n=1 \\ H^{n-1}(\Gamma, D) & n \geqslant 2\end{cases}
$$

Furthermore, if $M$ is torsion free, then

$$
H^{n}\left(\Gamma, X_{*}(D)\right)=\operatorname{Ext}_{\Gamma}^{n}(M, Z)
$$

There is an easy reduction to the case in which $\Gamma$ is finite. Then we have a spectral sequence

$$
H^{p}\left(\Gamma, \operatorname{Ext}_{\mathrm{Z}}^{q}(A, B)\right) \Rightarrow \operatorname{Ext}_{\mathrm{Z}[\Gamma]}^{n}(A, B)
$$

for any $\Gamma$-modules $A, B$ (see [C-E]). In particular, if $A$ is Z-projective or $B$ is Z-injective, then $H^{n}\left(\Gamma, \operatorname{Hom}_{\mathrm{Z}}(A, B)\right)=\operatorname{Ext}_{\mathrm{Z}[\Gamma]}^{n}(A, B)$ for all $n \geqslant 0$. Taking 
$A=M$ and $B=\mathrm{Z}$, we get the last statement of the lemma. Taking $A=M$ and $B=\mathrm{C}$, we see that $\operatorname{Ext}_{\mathrm{Z}[\Gamma]}^{n}(M, \mathrm{C})=0$ for $n>0$. Taking $A=M$ and $B=\mathrm{C}^{\times}$, we see that $\operatorname{Ext}_{\mathrm{Z}[\Gamma]}^{n}\left(M, \mathrm{C}^{\times}\right)=H^{n}(\Gamma, D)$. Now consider the long exact sequence obtained by applying $\operatorname{Ext}_{\bar{Z}[\Gamma]}^{\cdot}(M,-)$ to the exponential sequence $1 \rightarrow \mathrm{Z} \rightarrow \mathrm{C} \rightarrow \mathrm{C}^{\times}$ $\rightarrow 1$. We see immediately that $\operatorname{Ext}_{\Gamma}^{n}(M, Z)=H^{n-1}(\Gamma, D)$ for $n \geqslant 2$.

Now consider the case $n=1$. The group $\operatorname{Ext}_{\mathrm{Z}[\Gamma]}^{1}(M, \mathrm{Z})$ is equal to the cokernel of $\operatorname{Hom}_{\Gamma}(M, \mathrm{C}) \rightarrow \operatorname{Hom}_{\Gamma}\left(M, \mathrm{C}^{\times}\right) . \operatorname{But}_{\operatorname{Hom}_{\Gamma}}(M, \mathrm{C})=\operatorname{Lie}\left(D^{\Gamma}\right), \operatorname{Hom}_{\Gamma}\left(M, \mathrm{C}^{\times}\right)$ $=D^{\Gamma}$, and the homomorphism between the two is the exponential mapping. Since $\exp \left(\operatorname{Lie}\left(D^{\Gamma}\right)\right)$ is the identity component of $D^{\Gamma}$, we get the desired result.

Finally we consider the case $n=0$. We have $\operatorname{Ext}_{\mathrm{Z}[\Gamma]}^{0}(M, Z)=\operatorname{Hom}_{\mathrm{Z}}(M, Z)^{\Gamma}$ $=\left[\operatorname{Hom}_{\mathrm{C} \text {-groups }}\left(\mathrm{C}^{\times}, D\right)\right]^{\Gamma}=X_{*}(D)^{\Gamma}$.

2.3. Corollary. Let $1 \rightarrow D_{1} \rightarrow D_{2} \rightarrow D_{3} \rightarrow 1$ be an exact sequence of diagonalizable $\mathrm{C}$-groups with $\Gamma$-action. Then there is a natural long exact sequence

$$
\begin{aligned}
1 & \rightarrow X_{*}\left(D_{1}\right)^{\Gamma} \rightarrow X_{*}\left(D_{2}\right)^{\Gamma} \rightarrow X_{*}\left(D_{3}\right)^{\Gamma} \\
& \rightarrow \pi_{0}\left(D_{1}^{\Gamma}\right) \rightarrow \pi_{0}\left(D_{2}^{\Gamma}\right) \rightarrow \pi_{0}\left(D_{3}^{\Gamma}\right) \\
& \rightarrow H^{1}\left(\Gamma, D_{1}\right) \rightarrow H^{1}\left(\Gamma, D_{2}\right) \rightarrow H^{1}\left(\Gamma, D_{3}\right) \\
& \rightarrow H^{2}\left(\Gamma, D_{1}\right) \rightarrow H^{2}\left(\Gamma, D_{2}\right) \rightarrow H^{2}\left(\Gamma, D_{3}\right) \rightarrow \cdots .
\end{aligned}
$$

2.4. Let $G$ be a connected reductive group over $F$. We will construct isomorphisms

$$
\begin{aligned}
\pi_{0}\left(Z(\hat{G})^{\Gamma}\right) & =\operatorname{Pic} G, \\
H^{1}(F, Z(\hat{G})) & =\operatorname{Br}_{a} G,
\end{aligned}
$$

functorial in $G$ for normal homomorphisms $G_{1} \rightarrow G_{2}$ (see 1.8 for the definition of normal).

2.4.3. First consider groups $G$ for which $G_{\text {der }}$ is simply connected. From Lemma 6.9 and Corollary 6.11 of [Sa] we see that Pic $\bar{G}=0$, where $\bar{G}=G_{F_{\text {sep }} \text {, }}$, and that $H^{1}\left(F, X^{*}(G)\right)=\operatorname{Pic} G, H^{2}\left(F, X^{*}(G)\right)=\operatorname{Br}_{a} G$. Combining (1.8.2) and Lemma 2.2, we get the isomorphisms we want, and they are functorial in $G$. Before considering the general case, we need two lemmas.

2.4.4. Lemma. Let $G_{1} \rightarrow G_{2}$ be a homomorphism, and let $H_{i} \rightarrow G_{i}(i=1,2)$ be $z$-extensions. Then there exists a commutative diagram

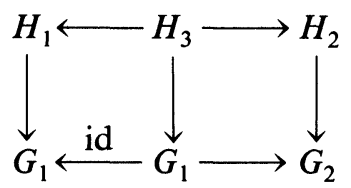

in which $H_{3} \rightarrow G_{1}$ is a z-extension. 
Take $H_{3}$ to be the fiber product of $H_{1}$ and $H_{2}$ over $G_{2}$. The surjectivity of $\mathrm{H}_{2} \rightarrow \mathrm{G}_{2}$ implies the surjectivity of $\mathrm{H}_{3} \rightarrow H_{1}$, which in turn implies the surjectivity of $H_{3} \rightarrow G_{1}$. The kernel of $H_{3} \rightarrow G_{1}$ is the product of the kernels of $H_{1} \rightarrow G_{1}$ and $H_{2} \rightarrow G_{2}$; in particular, it is an induced torus. It is clear that the kernel of $H_{3} \rightarrow G_{1}$ is central in $H_{3}$. The preceding statements imply that $H_{3}$ is connected reductive. Finally, since $H_{3} \rightarrow G_{1}$ factors through $H_{1} \rightarrow G_{1}$, the derived group of $\mathrm{H}_{3}$ is simply connected.

2.4.5. LemMA. Let $G$ be a connected reductive group over $F$. Then there exists a central extension $H \rightarrow G$ of $G$ such that $Z(\hat{H})$ is an induced torus. Let $\gamma: G_{1} \rightarrow G_{2}$ be a homomorphism, and let $\alpha_{i}: H_{i} \rightarrow G_{i}(i=1,2)$ be central extensions such that $Z\left(\hat{H}_{i}\right)(i=1,2)$ are induced tori. Then there exists a commutative diagram

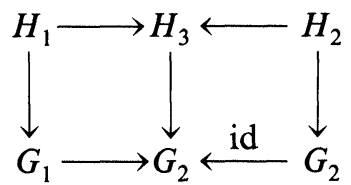

in which $H_{3} \rightarrow G_{2}$ is a central extension such that $Z\left(\hat{H}_{3}\right)$ is an induced torus.

First we show that central extensions $H \rightarrow G$ of the required type exist. If we had such a central extension, then we would get an embedding $Z(\hat{G}) \hookrightarrow Z(\hat{H})$. Conversely, given an embedding of $Z(\hat{G})$ in a torus $\hat{Z}$, we can find a central extension $H \rightarrow G$ with $Z(\hat{H})=\hat{Z}$ (see the proof of Proposition 3.1 of [M-S]). It is clear that we can choose $\hat{Z}$ to be induced.

Now we prove the existence of the diagram (2.4.5.1). Since the groups $Z\left(\hat{H}_{i}\right)$ $(i=1,2)$ are connected, the derived groups of $H_{i}(i=1,2)$ are simply connected. From the simple connectivity of $\left(H_{1}\right)_{\mathrm{der}}$ it follows that there exists a unique homomorphism $\beta:\left(H_{1}\right)_{\mathrm{der}} \rightarrow\left(H_{2}\right)_{\mathrm{der}}$ making the following diagram commute:

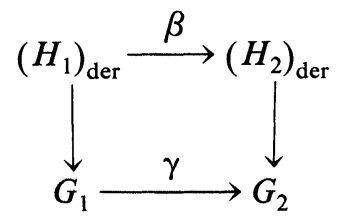

Let $C$ be the identity component of the center of $H_{1}$, and let $C_{0}=C \cap\left(H_{1}\right)_{\mathrm{der}}$. Then $H_{1}=\left(\left(H_{1}\right)_{\mathrm{der}} \times C\right) / C_{0}$. Now let $H_{3}=\left(H_{2} \times C\right) / C_{0}$, where $C_{0}$ is embedded in $H_{2} \times C$ by means of the homomorphism $c \mapsto\left(\beta(c), c^{-1}\right)$. We construct the homomorphisms needed in diagram (2.4.5.1) as follows:

$$
\begin{aligned}
H_{1} & \rightarrow H_{3} & \left(h_{1}, z\right) & \mapsto\left(\beta\left(h_{1}\right), z\right), \\
H_{2} & \rightarrow H_{3} & h_{2} & \mapsto\left(h_{2}, 1\right), \\
H_{3} & \rightarrow G_{2} & \left(h_{2}, z\right) & \mapsto \alpha_{2}\left(h_{2}\right) \cdot \gamma\left(\alpha_{1}(z)\right) .
\end{aligned}
$$


It is not hard to check that these homomorphisms are well defined, that diagram (2.4.5.1) commutes, and that $\mathrm{H}_{2} \rightarrow \mathrm{H}_{3}$ is an embedding. This last fact, together with the fact that $\left(\mathrm{H}_{2}\right)_{\text {der }}$ is simply connected, implies that $\left(\mathrm{H}_{3}\right)_{\mathrm{der}}$ is simply connected. By $(1.8 .3)$ we have $Z\left(\hat{H}_{i}\right)=\hat{D}_{i}$, where $D_{i}=H_{i} /\left(H_{i}\right)_{\mathrm{der}}(i=1,2,3)$, and it is easy to see that $D_{3}=D_{1} \times D_{2}$. Therefore $Z\left(\hat{H}_{3}\right)$ is an induced torus.

2.4.6. Now we finish the construction of the isomorphisms (2.4.1), (2.4.2), starting with (2.4.1). By Lemma 2.4 .5 we can find a central extension $H \rightarrow G$ such that $Z(\hat{H})$ is an induced torus. Let $Z$ denote the kernel of $H \rightarrow G$. We get an exact sequence $1 \rightarrow Z(\hat{G}) \rightarrow Z(\hat{H}) \rightarrow \hat{Z} \rightarrow 1$. Using Corollary 2.3 and the exact sequence $\cdots \rightarrow X^{*}(H)^{\Gamma} \rightarrow X^{*}(Z)^{\Gamma} \rightarrow \operatorname{Pic} G \rightarrow \operatorname{Pic} H \rightarrow \cdots$ of Corollary 6.11 of [Sa], we get a commutative diagram with exact rows

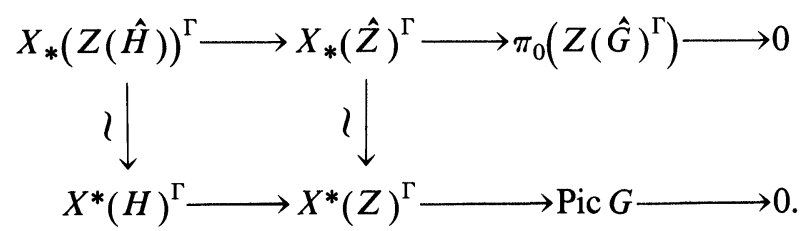

Note that $\pi_{0}(Z(\hat{H}))=0$ since $Z(\hat{H})$ is induced, and hence that Pic $H=0$ by 2.4.3. We define the isomorphism (2.4.1) to be the unique homomorphism making the diagram above commute. It is an easy application of Lemma 2.4 .5 to check that the isomorphisms we obtain are independent of the choice of $H \rightarrow G$ and are functorial for normal homomorphisms $G_{1} \rightarrow G_{2}$.

We will construct the isomorphism (2.4.2) in a similar way. However, this time we will use a $z$-extension $H \rightarrow G$. Again let $Z$ denote the kernel of $H \rightarrow G$. Since $Z$ is induced, we have $\pi_{0}\left(\hat{Z}^{\Gamma}\right)=0$. Then $\operatorname{Pic} Z=0$ by 2.4.3. Again using Corollary 2.3 and [Sa, Cor. 6.11], we get the following commutative diagram with exact rows:

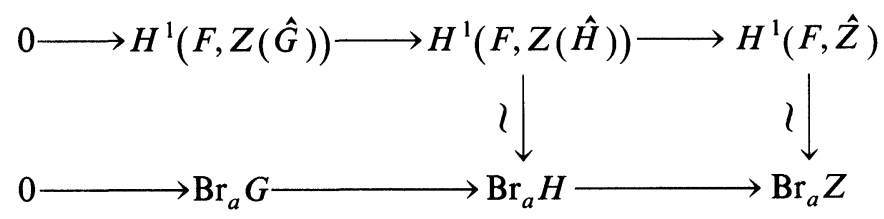

The vertical isomorphisms come from 2.4.3. We define the isomorphism (2.4.2) to be the unique homomorphism making the diagram above commute. It follows from Lemma 2.4.4 that the isomorphisms we obtain are independent of the choice of $z$-extension and are functorial for normal homomorphisms $G_{1} \rightarrow G_{2}$.

2.5. There is another way to look at the isomorphisms (2.4.1) and (2.4.2). The $F$-group $\mathrm{G}_{m}$ defines a sheaf of abelian groups for the étale topology on any $F$-variety. In particular, we have étale cohomology groups $H^{n}\left(G, \mathrm{G}_{m}\right)$ for any connected reductive group $G$ over $F$. Let $H^{n}\left(G, \mathrm{G}_{m}\right)_{e}$ denote the kernel of the homomorphism $H^{n}\left(G, \mathrm{G}_{m}\right) \rightarrow H^{n}\left(\operatorname{Spec} F, \mathrm{G}_{m}\right)$ induced by the identity element $e: \operatorname{Spec} F \rightarrow G$ of the group $G$. By a result of Rosenlicht [R] we have 
$H^{0}\left(G, \mathrm{G}_{m}\right)_{e}=X^{*}(G)^{\Gamma}$. It is well known that $H^{1}\left(G, \mathrm{G}_{m}\right)=\operatorname{Pic} G$, and from Hilbert's Theorem 90 it follows that $H^{1}\left(G, G_{m}\right)_{e}=$ Pic $G$ as well. By a result of Hoobler [Ho] we have $H^{2}\left(G, \mathrm{G}_{m}\right)=\operatorname{Br} G$, and therefore

$$
\operatorname{Br}_{a} G=\operatorname{ker}\left[H^{2}\left(G, \mathrm{G}_{m}\right)_{e} \rightarrow H^{2}\left(G_{F_{\text {sep }}}, \mathrm{G}_{m}\right)_{e}\right]
$$

Combining these facts with Lemma 2.2, we see that

$$
\operatorname{Ext}_{\Gamma}^{n}\left(X^{*}(Z(\hat{G})), \mathrm{Z}\right)=H^{n}\left(G, \mathrm{G}_{m}\right)_{e} \quad(n=0,1)
$$

and

$$
\operatorname{Ext}_{\Gamma}^{2}\left(X^{*}(Z(\hat{G})), Z\right)=\operatorname{ker}\left[H^{2}\left(G, \mathrm{G}_{m}\right)_{e} \rightarrow H^{2}\left(G_{F_{\text {sep }}}, \mathrm{G}_{m}\right)_{e}\right]
$$

It seems reasonable to expect that the same relationship holds between $\operatorname{Ext}_{\Gamma}^{n}\left(X^{*}(Z(\hat{G})), X_{*}(T)\right)$ and $H^{n}(G, T)_{e}$ for any $F$-torus $T$, but I have not checked this.

3. Review of Tate-Nakayama duality. Duality for tori plays an important role in the theory of $L$-indistinguishability. In this application of duality theory only the groups $H^{r}(F, T)$ are needed. Their behavior is simpler than that of the groups $H^{r}(L / F, T)$, where $L$ is a finite Galois extension of $F$. In this section we will review local and global duality for the groups $H^{r}(F, T)$.

3.1. Let $F$ be a nonarchimedean local field. The form of duality that we want can be found in a book by Shatz [S], and our presentation will follow his.

3.1.1. Let $A$ be any $\Gamma$-module. Then $H^{r}(F, A)=0$ for $r \geqslant 3$.

3.1.2. Let $T$ be an $F$-torus. Let $H^{0}\left(F, X^{*}(T)\right)^{c}$ denote the completion of $H^{0}\left(F, X^{*}(T)\right)$ in the topology of subgroups of finite index, and let $H^{0}(F, T)^{c}$ denote the completion of $H^{0}(F, T)=T(F)$ in the topology of open subgroups of finite index. The pairing $X^{*}(T) \times T \rightarrow \mathrm{G}_{m}$ induces cup-product pairings

$$
H^{r}\left(F, X^{*}(T)\right) \times H^{2-r}(F, T) \rightarrow H^{2}\left(F, \mathrm{G}_{m}\right)=\mathrm{Q} / \mathrm{Z}
$$

under which

(3.1.2.1) the compact group $H^{0}\left(F, X^{*}(T)\right)^{c}$ and discrete group $H^{2}(F, T)$ are dual,

(3.1.2.2) the finite groups $H^{1}\left(F, X^{*}(T)\right)$ and $H^{1}(F, T)$ are dual,

(3.1.2.3) the discrete group $H^{2}\left(F, X^{*}(T)\right)$ and compact group $H^{0}(F, T)^{c}$ are dual.

3.2. Let $F$ be an archimedean local field. Since $\Gamma$ is finite, we have the reduced groups $\tilde{H}^{r}(F, A)$ for any $\Gamma$-module $A$ and any $r \in Z$. Let $T$ be an $F$-torus. The 
pairing $X^{*}(T) \times T \rightarrow \mathrm{G}_{m}$ induces cup-product pairings

$$
\tilde{H}^{r}\left(F, X^{*}(T)\right) \times \tilde{H}^{2-r}(F, T) \rightarrow \tilde{H}^{2}\left(F, \mathrm{G}_{m}\right) \hookrightarrow \mathrm{Q} / \mathrm{Z}
$$

under which the finite groups $\tilde{H}^{r}\left(F, X^{*}(T)\right)$ and $\tilde{H}^{2-r}(F, T)$ are dual for all $r \in \mathbf{Z}$.

3.3. Let $F$ be any local field, archimedean or nonarchimedean, and let $T$ be an $F$-torus. Using Lemma 2.2, we get the following consequences of local duality:

$$
\text { the finite groups } \pi_{0}\left(\hat{T}^{\Gamma}\right) \text { and } H^{1}(F, T) \text { are dual, }
$$

the group $H^{1}(F, \hat{T})$ is canonically isomorphic to the group of continuous characters of finite order of $T(F)$ (this is part of the Langlands correspondence for $T$ ).

3.4. Let $F$ be a number field, and let $S$ denote the set of all places of $F$.

3.4.1. Let $A$ be a $\Gamma$-module. Then for $r \geqslant 3$ the canonical homomorphism $H^{r}(F, A) \rightarrow \bigoplus_{v \in S} H^{r}\left(F_{v}, A\right)$ is an isomorphism. Note that $H^{r}\left(F_{v}, A\right)=0$ for finite $v$, so that the direct sum could just as well be taken over the set of infinite places of $F$. Let $\operatorname{ker}^{r}(F, A)$ denote $\operatorname{ker}\left[H^{r}(F, A) \rightarrow \prod_{v \in S} H^{r}\left(F_{v}, A\right)\right]$. Then $\operatorname{ker}^{r}(F, A)=0$ unless $r=1,2$.

3.4.2. Let $T$ be an $F$-torus. Let $H^{0}\left(F, X^{*}(T)\right)^{c}$ denote the completion of $H^{0}\left(F, X^{*}(T)\right)$ in the topology of subgroups of finite index, and let $H^{0}\left(F, T\left(\mathrm{~A}_{\bar{F}}\right)\right.$ $/ T(\bar{F}))^{c}$ denote the completion of $H^{0}\left(F, T\left(\mathrm{~A}_{\bar{F}}\right) / T(\bar{F})\right)$ in the topology of open subgroups of finite index. The pairing $X^{*}(T) \times\left(T\left(\mathrm{~A}_{\bar{F}}\right) / T(\bar{F})\right) \rightarrow \mathrm{A}_{\bar{F}} / \bar{F}^{\times}$ induces cup-product pairings

$$
H^{r}\left(F, X^{*}(T)\right) \times H^{2-r}\left(F, T\left(\mathrm{~A}_{\bar{F}}\right) / T(\bar{F})\right) \rightarrow H^{2}\left(F, \mathrm{~A}_{\bar{F}}^{\times} / \bar{F}^{\times}\right)=\mathrm{Q} / \mathrm{Z}
$$

under which

(3.4.2.1) the compact group $H^{0}\left(F, X^{*}(T)\right)^{c}$ and the discrete group $H^{2}\left(F, T\left(\mathrm{~A}_{\bar{F}}\right) / T(\bar{F})\right)$ are dual,

(3.4.2.2) the finite groups $H^{1}\left(F, X^{*}(T)\right)$ and $H^{1}\left(F, T\left(\mathrm{~A}_{\bar{F}}\right) /\right.$ $T(\bar{F}))$ are dual,

(3.4.2.3) the discrete group $H^{2}\left(F, X^{*}(T)\right)$ and the compact group $H^{0}\left(F, T\left(\mathrm{~A}_{\bar{F}}\right) / T(\bar{F})\right)^{c}$ are dual.

3.4.3. Let $\operatorname{cok}^{r}(F, T)$ denote $\operatorname{cok}\left[H^{r}(F, T) \rightarrow H^{r}\left(F, T\left(\mathrm{~A}_{\bar{F}}\right)\right)\right]$. Note that $H^{r}(F$, $\left.T\left(\mathrm{~A}_{\bar{F}}\right)\right)=\bigoplus_{v \in S} H^{r}\left(F_{v}, T\right)$ for $r \geqslant 1$. By combining the cup-product pairing of 3.4.2 with the inclusion of $\operatorname{cok}^{2-r}(F, T)$ in $H^{2-r}\left(F, T\left(A_{\bar{F}}\right) / T(\bar{F})\right)$, we get a 
homomorphism

$$
H^{r}\left(F, X^{*}(T)\right) \rightarrow \operatorname{cok}^{2-r}(F, T)^{D},
$$

which can also be described as follows: from an element $\alpha \in H^{r}\left(F, X^{*}(T)\right)$ we get elements $\alpha_{v} \in H^{r}\left(F_{v}, X^{*}(T)\right)$, and then by means of the local pairings we get elements $\beta_{v} \in H^{2-r}\left(F_{v}, T\right)^{D}$, which fit together to give a character on $H^{2-r}\left(F, T\left(\mathrm{~A}_{\bar{F}}\right)\right)$, trivial on the image of $H^{2-r}(F, T)$. This homomorphism induces

$$
\text { an isomorphism } H^{0}\left(F, X^{*}(T)\right)^{c} \stackrel{\sim}{\longrightarrow} \operatorname{cok}^{2}(F, T)^{D},
$$

a surjection $H^{1}\left(F, X^{*}(T)\right) \rightarrow \operatorname{cok}^{1}(F, T)^{D}$, whose kernel is $\operatorname{ker}^{1}\left(F, X^{*}(T)\right)$,

a surjection $H^{2}\left(F, X^{*}(T)\right) \rightarrow\left[\pi_{0}(T(\mathrm{~A}) / T(F))\right]^{D}$, whose kernel is $\operatorname{ker}^{2}\left(F, X^{*}(T)\right)$.

3.4.4. The following three subgroups of $H^{r}(F, T)$ are the same:

(a) $\operatorname{ker}^{r}(F, T(\bar{F}))$,

(b) $\operatorname{ker}\left[H^{r}(F, T) \rightarrow \prod_{v \in S} H^{r}\left(F_{v}, T\right)\right]$,

(c) $\operatorname{ker}\left[H^{r}(F, T) \rightarrow H^{r}\left(F, T\left(\mathrm{~A}_{\bar{F}}\right)\right)\right]$.

We will denote this subgroup by $\operatorname{ker}^{r}(F, T)$. There is a pairing

$$
\operatorname{ker}^{r}\left(F, X^{*}(T)\right) \times \operatorname{ker}^{3-r}(F, T) \rightarrow \mathrm{Q} / \mathrm{Z}
$$

for $r=0,1,2,3$, defined in the same way as for finite $\Gamma$-modules [T1]. For $r=0,3$ the two groups being paired are trivial by 3.4.1. For $r=1,2$ the pairing is a perfect duality of finite groups.

3.4.5. Using Lemma 2.2, we can restate all of the results above in terms of $\hat{T}$, so that the duality theorems become statements about the duality of the cohomology of $T$ and $\hat{T}$. Here are two special cases:

(3.4.5.1) the groups $\operatorname{ker}^{1}(F, T)$ and $\operatorname{ker}^{1}(F, \hat{T})$ are dual finite abelian groups,

(3.4.5.2) there is a canonical surjection from $H^{1}(F, \hat{T})$ onto the group of continuous characters of finite order of $T(\mathrm{~A}) / T(F)$, and the kernel of this surjection is $\operatorname{ker}^{1}(F, \hat{T})$ (this is part of the global Langlands correspondence for $T$ ). 
4. Hasse principle. Let $F$ be a number field, and let $S$ denote the set of all places of $F$.

4.1. For an algebraic group $G$ over $F$ we will write $\operatorname{ker}^{1}(F, G)$ for the kernel of the Hasse map

$$
H^{1}(F, G) \rightarrow \prod_{v \in S} H^{1}\left(F_{v}, G\right)
$$

4.2. Let $G$ be a connected linear algebraic group over $F$, and assume that $G$ has no factor of type $E_{8}$. Sansuc [Sa] has shown that there is a canonical bijection between $\operatorname{ker}^{1}(F, G)$ and the dual of the finite abelian group

$$
\operatorname{ker}\left[\mathrm{Br}_{a} G \rightarrow \prod_{v \in S} \mathrm{Br}_{a}\left(G_{F_{v}}\right)\right]
$$

Now assume further that $G$ is reductive. Then from 2.4 we know that the group (4.2.1) is canonically isomorphic to $\operatorname{ker}^{1}(F, Z(\hat{G}))$ (here we are using the notation of 3.4.1). Combining this with Sansuc's result, we see that there is a canonical bijection between $\operatorname{ker}^{1}(F, G)$ and $\operatorname{ker}^{1}(F, Z(\hat{G}))^{D}$. Lemma 8.4 of [Sa] implies that in the case when $G$ is a torus this bijection is the negative of the bijection given by Tate-Nakayama duality (3.4.5.1). Renormalize all the bijections by replacing them with their negatives. Then for every connected reductive group $G$ with no $E_{8}$ factors there is a canonical bijection

$$
\operatorname{ker}^{1}(F, G) \rightarrow \operatorname{ker}^{1}(F, Z(\hat{G}))^{D}
$$

and these bijections satisfy the following two properties:

$$
\begin{aligned}
& \text { the bijections are functorial for normal homomor- } \\
& \text { phisms } G_{1} \rightarrow G_{2} \text {, }
\end{aligned}
$$

if $G$ is a torus, then the bijection is given by Tate-Nakayama duality (3.4.5.1).

4.3. In fact, the bijections (4.2.2) are characterized by properties (4.2.3) and (4.2.4). This is an immediate consequence of the following two lemmas.

4.3.1. Lemma. Let $G$ be a connected reductive group over $F$ having no $E_{8}$ factors. Assume that $G_{\mathrm{der}}$ is simply connected, and let $D=G / G_{\mathrm{der}}$. Then

(a) $\operatorname{ker}^{1}(F, \hat{D})=\operatorname{ker}^{1}(F, Z(\hat{G}))$,

(b) $\operatorname{ker}^{1}(F, G)=\operatorname{ker}^{1}(F, D)$.

Part (a) follows trivially from (1.8.3). The proof of (b) is essentially the same as the proof of Theorem 4.3 of [Sa]. Let $\beta$ be the map $\operatorname{ker}^{1}(F, G) \rightarrow \operatorname{ker}^{1}(F, D)$ induced by $G \rightarrow D$. First we prove that $\beta$ is injective. By a twisting argument it is 
enough to prove that $\operatorname{ker}(\beta)$ is trivial. We have an exact sequence

$$
\cdots \rightarrow D(F) \rightarrow H^{1}\left(F, G_{\text {sc }}\right) \rightarrow H^{1}(F, G) \rightarrow H^{1}(F, D),
$$

as well as the corresponding local sequences. Let $x \in \operatorname{ker}(\beta)$. Choose $y \in H^{1}\left(F, G_{\text {sc }}\right)$ such that $y$ maps to $x$. Since $x$ is locally trivial, the localization $y_{v}$ of $y$ at $v$ is in the image of $D\left(F_{v}\right)$ for all $v$. Since $D(F)$ is dense in $D\left(F \otimes_{\mathrm{Q}} \mathrm{R}\right)$, we can choose $z \in D(F)$ whose image $y^{\prime}$ in $H^{1}\left(F, G_{\text {sc }}\right)$ agrees with $y$ at the infinite places. Since $H^{1}\left(F_{v}, G_{\mathrm{sc}}\right)$ is trivial for finite $v$, the elements $y, y^{\prime}$ agree locally everywhere, hence are equal by the Hasse principle. Therefore $x$ is trivial.

Next we prove that $\beta$ is surjective. Let $x \in \operatorname{ker}^{1}(F, D)$. Choose a maximal $F$-torus $T$ of $G$ such that $T$ is elliptic at some finite place of $F$. Then $\operatorname{ker}^{2}\left(F, T_{\text {sc }}\right)=0$. It follows from the long exact cohomology sequence for

$$
1 \rightarrow T_{\mathrm{sc}} \rightarrow T \rightarrow D \rightarrow 1
$$

that $x$ is the image of some element $y \in H^{1}(F, T)$. Since $x$ is locally trivial, the localization $y_{v}$ of $y$ at $v$ is in the image of $H^{1}\left(F_{v}, T_{\mathrm{sc}}\right)$ for all $v$. Since $H^{1}\left(F, T_{\mathrm{sc}}\right)$ maps onto $\prod_{v \in S_{\infty}} H^{1}\left(F_{v}, T_{\mathrm{sc}}\right)$, where $S_{\infty}$ denotes the set of infinite places of $F$, we can modify $y$ by an element of $H^{1}\left(F, T_{\text {sc }}\right)$ in such a way that $y_{v}=1$ for all $v \in S_{\infty}$. Let $z$ be the image of $y$ in $H^{1}(F, G)$. It is obvious that $z$ maps to $x$, and we will see that $z \in \operatorname{ker}^{1}(F, G)$. Clearly $z$ is locally trivial at the infinite places. Consider a finite place $v$. Since $H^{1}\left(F_{v}, G_{\text {sc }}\right)=\{1\}$, the canonical map $H^{1}\left(F_{v}, G\right) \rightarrow H^{1}\left(F_{v}, D\right)$ has trivial kernel, and now the local triviality of $z$ at $v$ is obvious.

4.3.2. Lemma. Let $G$ be a connected reductive group over $F$, and let $H \rightarrow G$ be a z-extension. Then

(a) $\operatorname{ker}^{1}(F, Z(\hat{G}))=\operatorname{ker}^{1}(F, Z(\hat{H}))$,

(b) $\operatorname{ker}^{1}(F, H)=\operatorname{ker}^{1}(F, G)$.

Let $Z$ be the kernel of $H \rightarrow G$. First we prove (a). We have an exact sequence

$$
1 \rightarrow Z(\hat{G}) \rightarrow Z(\hat{H}) \rightarrow \hat{Z} \rightarrow 1,
$$

which yields a long exact sequence (see Corollary 2.3)

$$
\cdots \rightarrow \pi_{0}\left(\hat{Z}^{\Gamma}\right) \rightarrow H^{1}(F, Z(\hat{G})) \rightarrow H^{1}(F, Z(\hat{H})) \rightarrow H^{1}(F, \hat{Z}) \rightarrow \cdots
$$

globally and locally. Since $Z$ is induced, $\pi_{0}\left(\hat{Z}^{\Gamma}\right)=0$ both globally and locally, and furthermore $\operatorname{ker}^{1}(F, \hat{Z})=0$. It follows easily that $\operatorname{ker}^{1}(F, Z(\hat{G})) \rightarrow \operatorname{ker}^{1}(F$, $Z(\hat{H}))$ is an isomorphism.

Next we prove (b). We have an exact sequence

$$
H^{1}(F, Z) \rightarrow H^{1}(F, H) \rightarrow H^{1}(F, G) \rightarrow H^{2}(F, Z)
$$

globally and locally. Since $Z$ is induced, $H^{1}(F, Z)=0$ globally and locally, and 
furthermore $\operatorname{ker}^{2}(F, Z)=0$. It follows easily that $\operatorname{ker}^{1}(F, H) \rightarrow \operatorname{ker}^{1}(F, G)$ is an isomorphism.

4.4. Remark. Using the two lemmas above, together with Lemma 2.4.4, one can easily show the existence of bijections

$$
\operatorname{ker}^{1}(F, G) \rightarrow \operatorname{ker}^{1}(F, Z(\hat{G}))^{D}
$$

satisfying properties (4.2.3) and (4.2.4), without using $\mathrm{Br}_{a} G$. The first two lemmas force the definition of the bijection, and the other lemma shows that the bijections are well defined and functorial.

5. Tamagawa numbers. Let $F$ be a number field. For a connected reductive group $G$ over $F$ we will write $\tau(G)$ for the Tamagawa number of $G$ and $\tau_{1}(G)$ for the relative Tamagawa number $\tau(G) / \tau\left(G_{\mathrm{sc}}\right)$ of $G$. Of course we have $\tau(G)=\tau_{1}(G)$ if $G_{\text {sc }}$ satisfies Weil's conjecture that $\tau\left(G_{\mathrm{sc}}\right)=1$.

5.1. Sansuc [Sa] has shown that $\tau_{1}(G)=|\operatorname{Pic} G| \cdot\left|\operatorname{ker}^{1}(F, G)\right|^{-1}$ if $G$ has no $E_{8}$ factors. Using (2.4.1) and (4.2.2) we can rewrite this equality as

$$
\tau_{1}(G)=\left|\pi_{0}\left(Z(\hat{G})^{\Gamma}\right)\right| \cdot\left|\operatorname{ker}^{1}(F, Z(\hat{G}))\right|^{-1} .
$$

In this form it is not necessary to assume that $G$ has no $E_{8}$ factors (since the simply connected form of $E_{8}$ has trivial center, any $E_{8}$ factor of $G$ is indeed, as the name suggests, a direct factor of $G)$. For a torus $T$ we have $\pi_{0}\left(\hat{T}^{\Gamma}\right)=H^{1}(F$, $X^{*}(T)$ ), and we recover a formula of Ono [O]. For a semisimple group $G$ we have $Z(\hat{G})=X^{*}(C)$, where $C$ is the kernel of $G_{\mathrm{sc}} \rightarrow G$, and we again recover a formula of Ono [O].

5.2. The function $G \mapsto \tau_{1}(G)$ can be characterized by the following properties:

$$
\begin{gathered}
\tau_{1}(T)=\tau(T) \quad \text { for every torus } T \\
\tau_{1}(G)=\tau_{1}\left(G / G_{\mathrm{der}}\right) \quad \text { if } G_{\mathrm{der}} \text { is simply connected, } \\
\tau_{1}(G)=\tau_{1}(H) \cdot\left|\operatorname{cok}\left[X^{*}(H)^{\Gamma} \rightarrow X^{*}(Z)^{\Gamma}\right]\right| \quad \begin{array}{l}
\text { for any } z \text {-extension } \\
H \rightarrow G \text { with kernel } Z .
\end{array}
\end{gathered}
$$

It is trivial that $\tau_{1}$ satisfies the first property; that it satisfies the second two properties follows from Corollary 10.5 of Sansuc [Sa]. Note that $E_{8}$ factors again cause no trouble. The fact that $G \mapsto \tau_{1}(G)$ is uniquely characterized by the three properties above is obvious, since $z$-extensions exist for any $G$.

5.3. The formula (5.1.1) for $\tau_{1}(G)$ can be proved directly by checking that the function of $G$ defined by the right side of the formula satisfies the three properties that we used to characterize $\tau_{1}$. The first property is Ono's result on the Tamagawa numbers of tori. The second property is obvious from (1.8.3). The 
third property follows from part (a) of Lemma 4.3.2 and the exact sequence

$$
X_{*}(Z(\hat{H}))^{\Gamma} \rightarrow X_{*}(\hat{Z})^{\Gamma} \rightarrow \pi_{0}\left(Z(\hat{G})^{\Gamma}\right) \rightarrow \pi_{0}\left(Z(\hat{H})^{\Gamma}\right) \rightarrow 0
$$

obtained by applying Corollary 2.3 to the exact sequence

$$
1 \rightarrow Z(\hat{G}) \rightarrow Z(\hat{H}) \rightarrow \hat{Z} \rightarrow 1 \text {. }
$$

6. $H^{1}$ for $p$-adic groups. In this section we will calculate $H^{1}(F, G)$, where $G$ is a connected reductive group over a $p$-adic field $F$, in terms of $Z(\hat{G})$. First we need to construct certain central extensions.

6.1. Lemma. Let $G$ be a connected reductive group over a field $F$, let $S$ denote the identity component of the center of $G$, let $C=S \cap G_{\mathrm{der}}$, and let $C^{\prime}$ denote the inverse image of $C$ under $p: G_{\mathrm{sc}} \rightarrow G_{\mathrm{der}}$. Let $i: C^{\prime} \rightarrow Z$ be an embedding of $C^{\prime}$ into an $F$-torus $Z$. Then there exists a central extension $G^{\prime}$ of $G$ by $Z$ such that $G_{\mathrm{der}}^{\prime}$ is simply connected.

If $\operatorname{char}(F) \neq 0$, the statement of the lemma must be interpreted with some care: $C=S \times{ }_{G} G_{\text {der }}$, and therefore $C$ may be a nonreduced scheme. To construct $G^{\prime}$ we first define $j: C^{\prime} \rightarrow G_{\mathrm{sc}} \times S \times Z$ by $j\left(c^{\prime}\right)=\left(c^{\prime}, p\left(c^{\prime}\right), i\left(c^{\prime}\right)\right)$, and then we put $G^{\prime}=G_{\text {sc }} \times S \times Z / j\left(C^{\prime}\right)$. Define $\alpha: G^{\prime} \rightarrow G$ by $\alpha(g, s, z)=p(g)$. $s^{-1}$. It is easy to see that $\alpha$ is well defined and surjective. Furthermore, $\operatorname{ker} \alpha$ is isomorphic to $Z$ and is central in $G^{\prime}$. We will show that $G_{\mathrm{der}}^{\prime}$ is simply connected by checking that the homomorphism $G_{\mathrm{sc}} \rightarrow G^{\prime}$ defined by $g \mapsto(g, 1,1)$ is an embedding. Clearly $\operatorname{ker}\left(G_{\mathrm{sc}} \rightarrow G^{\prime}\right)$ is a subgroup of $\operatorname{ker}\left(G_{\mathrm{sc}} \rightarrow G\right)$, which is in turn a subgroup of $C^{\prime}$. This reduces us to showing that the homomorphism $C^{\prime} \rightarrow G^{\prime}$ defined by $c^{\prime} \mapsto\left(c^{\prime}, 1,1\right)$ has trivial kernel. But this homomorphism factors through the subgroup $C^{\prime} \times S \times Z / j\left(C^{\prime}\right)$ of $G^{\prime}$. Call this subgroup $S^{\prime}$. If we compose $C^{\prime} \rightarrow S^{\prime}$ with the homomorphism $S^{\prime} \rightarrow Z$ defined by $\left(c^{\prime}, s, z\right) \mapsto i\left(c^{\prime}\right)$. $z^{-1}$, we get the embedding $i$. Therefore $C^{\prime} \rightarrow S^{\prime}$ has trivial kernel.

6.2. Lemma. Let $F$ be a $p$-adic field. Then any finite diagonalizable $F$-group $A$ can be embedded in an anisotropic $F$-torus.

Let $m$ be the order of $A$. Let $K$ be a finite Galois extension of $F$ such that the action of $\Gamma$ on $X^{*}(A)$ factors through $\operatorname{Gal}(K / F)$. Then $A$ can be embedded in a finite product of copies of $T$, where $T=\operatorname{Res}_{K / F} \mathrm{G}_{m}$. Therefore it suffices to treat the case $A=T_{m}$, where $T_{m}=\left\{t \in T: t^{m}=1\right\}$. Let $L$ be an extension of $K$ of degree $m$ (for instance, the unramified extension of degree $m$ ). There is a natural embedding of $T$ in $\operatorname{Res}_{L / F} \mathrm{G}_{m}$. The restriction of the norm homomorphism $N: \operatorname{Res}_{L / F} \mathrm{G}_{m} \rightarrow \mathrm{G}_{m}$ to the subgroup $T$ is the $m$ th power of the norm homomorphism $T \rightarrow \mathrm{G}_{m}$. Therefore $T_{m}$ is contained in ker $N$, an anisotropic $F$-torus.

6.3. Let $F$ be a $p$-adic field. For any torus $T$ over $F$ we have a canonical 
isomorphism

$$
H^{1}(F, T) \stackrel{\sim}{\longrightarrow}\left[\pi_{0}\left(\hat{T}^{\Gamma}\right)\right]^{D}
$$

obtained from local duality (3.3.1). Now consider the two functors $G \mapsto H^{1}(F, G)$ and $G \mapsto\left[\pi_{0}\left(Z(\hat{G})^{\Gamma}\right)\right]^{D}$ from the category of connected reductive $F$-groups and normal homomorphisms to the category of sets.

6.4. Proposition. There is a unique extension of (6.3.1) to an isomorphism of functors

$$
H^{1}(F, G) \stackrel{\sim}{\longrightarrow}\left[\pi_{0}\left(Z(\hat{G})^{\Gamma}\right)\right]^{D}
$$

We extend the isomorphism of functors in two stages. At the first stage we extend it to groups $G$ such that $G_{\text {der }}$ is simply connected. Consider such a group $G$, and let $D=G / G_{\text {der. }}$. In view of the isomorphism $Z(\hat{G})=\hat{D}$ the existence and uniqueness in the first stage of the extension will be obvious once we show that

$$
H^{1}(F, G)=H^{1}(F, D) \text {. }
$$

Using a twisting argument plus the vanishing of $H^{1}$ for simply connected $p$-adic groups [Kn], we see that $H^{1}(F, G) \rightarrow H^{1}(F, D)$ is injective. Let $T$ be an elliptic maximal $F$-torus of $G$; such tori exist by a result of Kneser [Kn]. The sequence $1 \rightarrow T_{\mathrm{sc}} \rightarrow T \rightarrow D \rightarrow 1$ is exact, and from it we get a long exact sequence

$$
\cdots \rightarrow H^{1}(F, T) \rightarrow H^{1}(F, D) \rightarrow H^{2}\left(F, T_{\mathrm{sc}}\right) \rightarrow \cdots .
$$

But $T_{\text {sc }}$ is anisotropic, and therefore $H^{2}\left(F, T_{\text {sc }}\right)=0$ by local duality. This proves the surjectivity of $H^{1}(F, T) \rightarrow H^{1}(F, D)$, and hence the surjectivity of $H^{1}(F, G)$ $\rightarrow H^{1}(F, D)$.

At the second stage we extend (6.4.1) to all groups. Given a group $G$, we choose a central extension $H \rightarrow G$ such that

(a) the kernel $Z$ of $H \rightarrow G$ is an anisotropic $F$-torus,

(b) $H_{\text {der }}$ is simply connected.

The existence of such an extension is guaranteed by Lemmas 6.1 and 6.2. We have the following commutative diagram with exact rows

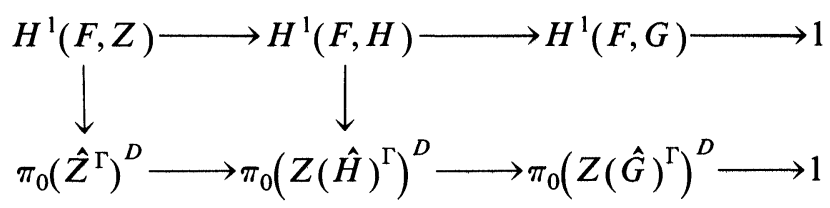

in which the vertical arrows are the bijections coming from the first stage. The trivial groups at the right-hand ends of the rows are $H^{2}(F, Z)$ (trivial by local 
duality since $Z$ is anisotropic) and $\left[X_{*}(\hat{Z})^{\Gamma}\right]^{D}$ (see Corollary $2.3 ; X_{*}(\hat{Z})^{\Gamma}$ is trivial since $Z$ is anisotropic). The fibers of $H^{1}(F, H) \rightarrow H^{1}(F, G)$ are the orbits for the action of $H^{1}(F, Z)$ on $H^{1}(F, H)$. Therefore there exists a unique bijection $H^{1}(F, G) \rightarrow \pi_{0}\left(Z(\hat{G})^{\Gamma}\right)^{D}$ that makes the diagram above commute. This proves the uniqueness of the extension (6.4.1). It is easy to check, using a variant of Lemma 2.4.4, that the bijections we obtain are independent of the choice of $H \rightarrow G$ and are functorial in $G$. This proves the existence of the extension (6.4.1).

6.5. Remark. Suppose that $G$ is semisimple, and let $C$ denote the kernel of $G_{\mathrm{sc}} \rightarrow G$. The exact sequence $1 \rightarrow C \rightarrow G_{\mathrm{sc}} \rightarrow G \rightarrow 1$ induces a map $H^{1}(F, G)$ $\rightarrow H^{2}(F, C)$. Kneser [Kn] has shown that this map is bijective. By local duality for finite groups we have $H^{2}(F, C)=\left[X^{*}(C)^{\Gamma}\right]^{D}$, and since $X^{*}(C)=Z(\hat{G})$, we see that Kneser's bijection can be reformulated as a bijection

$$
H^{1}(F, G) \stackrel{\sim}{\longrightarrow} \pi_{0}\left(Z(\hat{G})^{\Gamma}\right)^{D}
$$

It is not hard to check that this bijection is the same as the one provided by Proposition 6.4. We choose a central extension $H \rightarrow G$ as in the proof of Proposition 6.4 and write $D$ for $H / H_{\text {der }}$ and $Z$ for $\operatorname{ker}(H \rightarrow G)$. The exact sequence

$$
1 \rightarrow C \rightarrow Z \times G_{\mathrm{sc}} \rightarrow H \rightarrow 1
$$

maps to both of the exact sequences

$$
\begin{aligned}
& 1 \rightarrow C \rightarrow G_{\mathrm{sc}} \rightarrow G \rightarrow 1, \\
& 1 \rightarrow C \rightarrow Z \rightarrow D \rightarrow 1 .
\end{aligned}
$$

Using this fact, we see that in order to check the agreement of the two bijections, it is enough to check the commutativity of the diagram

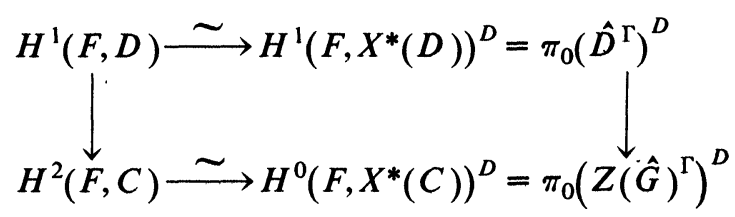

in which the vertical maps come from the exact sequence

$$
1 \rightarrow C \rightarrow Z \rightarrow D \rightarrow 1 \text {. }
$$

This is easy.

6.6. Remark. Using (2.4.1), we can put the bijection (6.4.1) into the following form:

$$
H^{1}(F, G) \stackrel{\sim}{\longrightarrow}(\operatorname{Pic} G)^{D}
$$


This is reminiscent of Tate's result [T1] on $H^{1}(F, A)$ for an abelian variety $A$ over a $p$-adic field $F$ :

$$
H^{1}(F, A) \stackrel{\sim}{\longrightarrow}\left(A^{\prime}(F)\right)^{D}
$$

where $A^{\prime}=\operatorname{Pic}^{0}(A)$, the dual abelian variety.

7. Review of endoscopic groups. In this section, $F$ is a local or global field of characteristic 0 , and $G$ is a connected reductive group over $F$.

7.1. An endoscopic datum for $G$ is a pair $(s, \rho)$, consisting of a semisimple element $s$ of $\hat{G} / Z(\hat{G})$ and a homomorphism $\rho: \Gamma \rightarrow \operatorname{Out}\left(\hat{G}_{s}^{0}\right)$, satisfying properties (7.1.1) and (7.1.2) below. We will write $\hat{H}$ for $\hat{G}_{s}^{0}$.

For $\sigma \in \Gamma$ the element $\rho(\sigma) \in \operatorname{Out}(\hat{H})$ is induced by an element of $\operatorname{Norm}_{{ }_{L} G}(\hat{H})$ whose image under the canonical homomorphism ${ }^{L} G \rightarrow \Gamma$ is $\sigma$.

Note that $\rho$ induces an action of $\Gamma$ on $Z(\hat{H})$. Moroever, the inclusion of $Z(\hat{G})$ in $Z(\hat{H})$ is a $\Gamma$-map (the action on $Z(\hat{G})$ comes from the action on $\hat{G}$ ). The exact sequence

$$
1 \rightarrow Z(\hat{G}) \rightarrow Z(\hat{H}) \rightarrow Z(\hat{H}) / Z(\hat{G}) \rightarrow 1
$$

gives us a long exact sequence (Corollary 2.3)

$$
\cdots \rightarrow \pi_{0}\left(Z(\hat{H})^{\Gamma}\right) \rightarrow \pi_{0}\left([Z(\hat{H}) / Z(\hat{G})]^{\Gamma}\right) \rightarrow H^{1}(F, Z(\hat{G})) \rightarrow \cdots .
$$

We define $\Re(s, \rho)$ to be the subgroup of $\pi_{0}\left([Z(\hat{H}) / Z(\hat{G})]^{\Gamma}\right)$ consisting of all elements whose image in $H^{1}(F, Z(\hat{G}))$ is

(a) trivial if $F$ is local,

(b) locally trivial if $F$ is global.

Now we can state the second condition on $(s, \rho)$. Note that $s \in Z(\hat{H}) / Z(\hat{G})$.

(7.1.2) The element $s \in Z(\hat{H}) / Z(\hat{G})$ is fixed by $\Gamma$, and its image in $\pi_{0}\left([Z(\hat{H}) / Z(\hat{G})]^{\Gamma}\right)$ belongs to $\Re(s, \rho)$.

7.2. Let $\left(s_{i}, \rho_{i}\right)(i=1,2)$ be endoscopic data for $G$ and write $\hat{H}_{i}$ for $\hat{G}_{s_{i}}^{0}$. An isomorphism from $\left(s_{1}, \rho_{1}\right)$ to $\left(s_{2}, \rho_{2}\right)$ is an element $g \in \hat{G}$ such that

$$
\operatorname{Int}(g)\left(\hat{H}_{1}\right)=\hat{H}_{2} \text {. }
$$

$\rho_{2}=\alpha \circ \rho_{1}$, where $\alpha$ denotes the isomorphism $\operatorname{Out}\left(\hat{H}_{1}\right)$

$\rightarrow \operatorname{Out}\left(\hat{H}_{2}\right)$ induced by $\operatorname{Int}(g)$,

$$
\operatorname{Int}(g)\left(s_{1}\right) \text { and } s_{2} \text { have the same image in } \Re\left(s_{2}, \rho_{2}\right) \text {. }
$$


We will write $\operatorname{Aut}(s, \rho)$ for the group of automorphisms of $(s, \rho)$. It is easy to see that $\operatorname{Aut}(s, \rho)$ is an algebraic subgroup of $\hat{G}$ with identity component $\hat{H}$. We will write $\Lambda(s, \rho)$ for the quotient $\operatorname{Aut}(s, \rho) / \hat{H}$.

7.3. We say that an endoscopic datum $(s, \rho)$, with associated group $\hat{H}=\hat{G}_{s}^{0}$, is elliptic, if $\left[Z(\hat{H})^{\mathrm{\Gamma}}\right]^{0} \subset Z(\hat{G})$. For elliptic endoscopic data the third condition in the definition of isomorphism can be replaced by

$$
\operatorname{Int}(g)\left(s_{1}\right)=s_{2} .
$$

7.4. An endoscopic triple for $G$ is a triple $(H, s, \eta)$, consisting of a quasi-split connected reductive $F$-group $H$ (which we will refer to as an endoscopic group for $G)$, an element $s \in Z(\hat{H})$, and an embedding $\eta: \hat{H} \rightarrow \hat{G}$ of $\mathrm{C}$ groups, satisfying the following three conditions:

$$
\eta(\hat{H})=\hat{G}_{\eta(s)}^{0} .
$$

The $\hat{G}$-conjugacy class of $\eta$ is fixed by $\Gamma$.

We can use $\eta$ to regard $Z(\hat{G})$ as a subgroup of $Z(\hat{H})$. By (7.4.2) the $\Gamma$-actions on $Z(\hat{G})$ and $Z(\hat{H})$ are compatible. Thus we can define a subgroup $\Re(H / F)$ of $\pi_{0}\left([Z(\hat{H}) / Z(\hat{G})]^{\Gamma}\right)$ as in 7.1 . Now we can state the third condition.

The image of $s$ in $Z(\hat{H}) / Z(\hat{G})$ is fixed by $\Gamma$, and its image in $\pi_{0}\left([Z(\hat{H}) / Z(\hat{G})]^{\Gamma}\right)$ belongs to $\Re(H / F)$.

7.5. Let $\left(H_{i}, s_{i}, \eta_{i}\right)(i=1,2)$ be endoscopic triples for $G$. An isomorphism from $\left(H_{1}, s_{1}, \eta_{1}\right)$ to $\left(H_{2}, s_{2}, \eta_{2}\right)$ is an $F$-isomorphism $\alpha: H_{1} \rightarrow H_{2}$ satisfying the following two conditions:

$\eta_{1} \circ \hat{\alpha}$ and $\eta_{2}$ are $\hat{G}$-conjugate. This condition makes sense because $\hat{\alpha}$ is well-defined up to $\hat{H}_{1}$-conjugacy. Furthermore, one consequence of this condition is that $\hat{\alpha}$ induces a canonical isomorphism $\Re\left(H_{2} / F\right)$ $\stackrel{\sim}{\rightarrow}\left(H_{1} / F\right)$.

(7.5.2) The elements of $\mathscr{\Re}\left(H_{i} / F\right)$ defined by $s_{i}$ correspond under $\AA\left(H_{2} / F\right) \stackrel{\sim}{\rightarrow} \Re\left(H_{1} / F\right)$.

We will write $\operatorname{Aut}(H, s, \eta)$ for the group of automorphisms of $(H, s, \eta)$. Clearly $H_{\mathrm{ad}}(F)$ is a normal subgroup of $\operatorname{Aut}(H, s, \eta)$. We will write $\Lambda(H, s, \eta)$ for the quotient $\operatorname{Aut}(H, s, \eta) / H_{\mathrm{ad}}(F)$.

7.6. An endoscopic triple $(H, s, \eta)$ determines an endoscopic datum $(\eta(s), \rho)$, where $\rho$ is the composition

$$
\Gamma \longrightarrow \operatorname{Aut}(\hat{H}) \stackrel{\sim}{\longrightarrow} \operatorname{Aut}\left(\hat{G}_{\eta(s)}^{0}\right) \longrightarrow \operatorname{Out}\left(\hat{G}_{\eta(s)}^{0}\right),
$$


and every endoscopic datum arises from some endoscopic triple. Let $\left(H_{i}, s_{i}, \eta_{i}\right)$ $(i=1,2)$ be endoscopic triples, and let $\left(\eta\left(s_{i}\right), \rho_{i}\right)(i=1,2)$ be the corresponding endoscopic data. There is a canonical bijection from the set of isomorphisms from $\left(H_{1}, s_{1}, \eta_{1}\right)$ to $\left(H_{2}, s_{2}, \eta_{1}\right)$, taken modulo inner automorphisms of $H_{2}$, to the set of isomorphisms from $\left(\eta\left(s_{1}\right), \rho_{1}\right)$ to $\left(\eta\left(s_{2}\right), \rho_{2}\right)$, taken modulo $\hat{G}_{\eta\left(s_{2}\right)}^{0}$. Thus there is a bijection from the set of isomorphism classes of endoscopic triples to the set of isomorphism classes of endoscopic data. Moreover, there is a canonical isomorphism

$$
\Lambda(H, s, \eta) \stackrel{\sim}{\longrightarrow} \Lambda(\eta(s), \rho) .
$$

We will say that $(H, s, \eta)$ is elliptic if $(\eta(s), \rho)$ is elliptic.

7.7. There are some related definitions from [L1, L3] that we need to recall. Let $T$ be a maximal $F$-torus of $G$. We denote by $\mathscr{D}(T / F)$ the set $\operatorname{ker}\left[H^{1}(F, T) \rightarrow H^{1}(F, G)\right]$. This set is in one-to-one correspondence with the set of $G(F)$-conjugacy classes of embeddings $j: T \rightarrow G$ such that $j$ is $G(\bar{F})$-conjugate to the inclusion $T \rightarrow G$. We denote by $\mathscr{E}(T / F)$ the image of $H^{1}\left(F, T_{\text {sc }}\right)$ in $H^{1}(F, T)$. It is always true that $\mathscr{D}(T / F)$ is contained in $\mathbb{E}(T / F)$, and the two sets are equal if $F$ is $p$-adic.

In the global case we denote by $\mathfrak{D}(T / \mathrm{A})$ the set

$$
\operatorname{ker}\left[H^{1}\left(F, T\left(\mathrm{~A}_{\bar{F}}\right)\right) \rightarrow H^{1}\left(F, G\left(\mathrm{~A}_{\bar{F}}\right)\right)\right]=\bigoplus_{v} \mathfrak{D}\left(T / F_{v}\right)
$$

and by $\&(T / A)$ the group

$$
\operatorname{im}\left[H^{1}\left(F, T_{\mathrm{sc}}\left(\mathrm{A}_{\bar{F}}\right)\right) \rightarrow H^{1}\left(F, T\left(\mathrm{~A}_{\bar{F}}\right)\right)\right]=\bigoplus_{v} \mathbb{E}\left(T / F_{v}\right),
$$

where the sums are taken over the set of all places of $F$ (it makes sense to take the direct sum of the sets $\mathfrak{D}\left(T / F_{v}\right)$ since they are pointed sets). There are obvious maps $\mathfrak{D}(T / F) \rightarrow \mathfrak{D}(T / \mathrm{A})$ and $\mathfrak{E}(T / F) \rightarrow \mathfrak{E}(T / \mathrm{A})$.

We also need to define groups $\Re(T / F)$. From the definition of the $L$-group we see that $Z(\hat{G})$ may be identified with a $\Gamma$-submodule of $\hat{T}$. The torus $\hat{T} / Z(\hat{G})$ is dual to the torus $T_{\text {sc }}$. From the exact sequence

$$
1 \rightarrow Z(\hat{G}) \rightarrow \hat{T} \rightarrow \hat{T} / Z(\hat{G}) \rightarrow 1
$$

we get a long exact sequence (Corollary 2.3)

$$
\cdots \rightarrow \pi_{0}\left(Z(\hat{T})^{\Gamma}\right) \rightarrow \pi_{0}\left([\hat{T} / Z(\hat{G})]^{\Gamma}\right) \rightarrow H^{1}(F, Z(\hat{G})) \rightarrow \cdots
$$

We define $\Re(T / F)$ to be the subgroup of $\pi_{0}\left([\hat{T} / Z(\hat{G})]^{\Gamma}\right)$ consisting of all elements whose image in $H^{1}(F, Z(\hat{G}))$ is

(a) trivial if $F$ is local,

(b) locally trivial if $F$ is global. 
If $F$ is global and $v$ is a place of $F$, there is a natural homomorphism $\Re(T / F) \rightarrow \Re\left(T / F_{v}\right)$. Note the similarity with the definition of $\Re(H / F)$ for an endoscopic group $H$. Our definition of $\Re(T / F)$ agrees with that of [L3] when $T$ is elliptic, but is slightly different otherwise, because Langlands does not divide by the identity component of $[\hat{T} / Z(\hat{G})]^{\Gamma}$. This difference is not important; dividing by the identity component is simply a technical convenience.

7.8. It is useful to know how $\mathfrak{D}(T / F),(\mathbb{E}(T / F), \Re(T / F)$ behave under $z$-extensions. In fact, the behavior is as simple as possible. Let $G^{\prime} \rightarrow G$ be a $z$-extension, let $T$ be maximal $F$-torus of $G$, and let $T^{\prime}$ be the inverse image of $T$ in $G^{\prime}$. Then the canonical maps $\mathfrak{D}\left(T^{\prime} / F\right) \rightarrow \mathfrak{D}(T / F), \mathbb{E}\left(T^{\prime} / F\right) \rightarrow \mathfrak{E}(T / F)$, $\Re(T / F) \rightarrow \Re\left(T^{\prime} / F\right)$ are all bijections. The proof is not difficult and will be left to the reader.

7.9. In the local case Tate-Nakayama duality (3.3.1) provides us with an isomorphism

$$
\Re(T / F)=\mathfrak{E}(T / F)^{D},
$$

since the dual of the image of $H^{1}\left(F, T_{\mathrm{sc}}\right) \rightarrow H^{1}(F, T)$ is equal to the image of $\pi_{0}\left(\hat{T}^{\Gamma}\right) \rightarrow \pi_{0}\left([\hat{T} / Z(\hat{G})]^{\Gamma}\right)$. In the global case Tate-Nakayama duality (3.4.3.2) provides us with a surjection

$$
\Re(T / F) \rightarrow[\Subset(T / \mathrm{A}) / \operatorname{im}(\Subset(T / F))]^{D},
$$

whose kernel consists of all the elements of $\mathscr{\Re}(T / F)$ which have trivial image in $\Re\left(T / F_{v}\right)$ for every place $v$ of $F$.

8. $\iota(G, H)$. Let $F$ be a number field, and let $G$ be a connected reductive group over $F$. To each endoscopic triple $(H, s, \eta)$ for $G$, Langlands [L3] has associated a constant $\iota(G, H)$, needed to stabilize the trace formula for $G$. This constant depends on $s$ and $\eta$, as well as $H$, although the notation may suggest otherwise. First we will recall the definition of $\iota(G, H)$, and then in 8.3 we will find a new expression for it.

8.1. We will write $\lambda(H, s, \eta)$, or just $\lambda$, for the order of the group $\Lambda(H, s, \eta)$ (see 7.5 and 7.6.1); $\lambda$ depends only on the isomorphism class of $(H, s, \eta)$.

8.2. Let $T$ be a maximal $F$-torus of $G$. Following Langlands [L3], we define a number $\iota(F, T, G)$ by putting

$$
\iota(F, T, G)=|\operatorname{ker}[\Subset(T / F) \rightarrow \Subset(T / \mathrm{A})]| \cdot|\Re(T / F)|^{-1} .
$$

Let $(H, s, \eta)$ be an elliptic endoscopic triple for $G$, and let $\psi: G^{*} \rightarrow G$ be an inner twisting, where $G^{*}$ is a quasi-split inner form of $G$. Any maximal $F$-torus $T_{H}$ of $H$ gives rise to a maximal $F$-torus $T_{G^{*}}$ of $G^{*}$, well-defined up to stable conjugacy (see [L3]). Using the fact that $(H, s, \eta)$ is elliptic, we see that $T_{H}$ is elliptic in $H$ if and only if $T_{G^{*}}$ is elliptic in $G^{*}$. In Lemma 8.6 of [L3] Langlands shows that the 
numbers

$$
\iota\left(F, T_{G^{*}}, G^{*}\right) \cdot \iota\left(F, T_{H}, H\right)^{-1}
$$

are all equal, as long at $T_{H}$ is elliptic, and he then defines $\iota(G, H)$ to be $\alpha \cdot \lambda^{-1}$, where $\alpha$ is the common value of the numbers (8.2.2).

8.3. Let $(H, s, \eta)$ be an elliptic endoscopic triple for $G$, and let $\lambda=\lambda(H, s, \eta)$. As in Section 5 we will use $\tau_{1}(G)$ to denote the relative Tamagawa number of $G$.

8.3.1 TheOREM. The constant $\iota(G, H)$ is equal to $\tau_{1}(G) \cdot \tau_{1}(H)^{-1} \cdot \lambda^{-1}$.

This equality is an immediate consequence of the following lemma (note that $\left.\tau_{1}\left(G^{*}\right)=\tau_{1}(G)\right)$.

8.3.2. Lemma. Let $T$ be a maximal F-torus of $G$. Let $T_{\mathrm{sc}}$ denote the corresponding maximal torus of $G_{\mathrm{sc}}$. Then

$$
\iota(F, T, G)=\tau_{1}(G) \cdot \tau_{1}(T)^{-1} \cdot\left|\operatorname{cok}\left[X^{*}(T)^{\Gamma} \rightarrow X^{*}\left(T_{\mathrm{sc}}\right)^{\Gamma}\right]\right|^{-1} .
$$

In particular, if $T$ is elliptic in $G$, then

$$
\iota(F, T, G)=\tau_{1}(G) \cdot \tau_{1}(T)^{-1} .
$$

By choosing a $z$-extension of $G$ and using (5.2.3) and 7.8, we reduce to the case in which $G_{\text {der }}$ is simply connected. Next we apply Corollary 2.3 to the exact sequence

$$
1 \rightarrow Z(\hat{G}) \rightarrow \hat{T} \rightarrow \hat{T}_{\mathrm{sc}} \rightarrow 1 .
$$

This gives us an exact sequence

$$
1 \rightarrow A \rightarrow \pi_{0}\left(Z(\hat{G})^{\Gamma}\right) \rightarrow \pi_{0}\left(\hat{T}^{\Gamma}\right) \rightarrow \pi_{0}\left(\hat{T}_{\text {sc }}^{\Gamma}\right) \rightarrow H^{1}(F, Z(\hat{G})) \rightarrow H^{1}(F, \hat{T}),
$$

where $A=\operatorname{cok}\left[X^{*}(T)^{\Gamma} \rightarrow X^{*}\left(T_{\mathrm{sc}}\right)^{\Gamma}\right]$. Using the definition of $\Re(T / F)$ and the exact sequence above, we get an exact sequence

$1 \rightarrow A \rightarrow \pi_{0}\left(Z(\hat{G})^{\Gamma}\right) \rightarrow \pi_{0}\left(\hat{T}^{\Gamma}\right) \rightarrow \Re(T / F) \rightarrow \operatorname{ker}^{1}(F, Z(\hat{G})) \rightarrow \operatorname{ker}^{1}(F, \hat{T}) \rightarrow B \rightarrow 1$, where $B=\operatorname{cok}\left[\operatorname{ker}^{1}(F, Z(\hat{G})) \rightarrow \operatorname{ker}^{1}(F, \hat{T})\right]$. Using (5.1.1), we see that

$$
|B| \cdot|\Re(T / F)|^{-1}=\tau_{1}(G) \cdot \tau_{1}(T)^{-1} \cdot|A|^{-1} .
$$

Since $G_{\mathrm{der}}$ is simply connected, we have $Z(\hat{G})=\hat{D}$, where $D=G / G_{\mathrm{der}}$, and by duality (3.4.5.1) the group $B$ is dual to

$$
\operatorname{ker}\left[\operatorname{ker}^{1}(F, T) \rightarrow \operatorname{ker}^{1}(F, D)\right]
$$


which, again because $G_{\text {der }}$ is simply connected, is equal to

$$
\operatorname{ker}[\mathfrak{E}(T / F) \rightarrow \mathfrak{E}(T / \mathrm{A})] .
$$

Combining this fact with (8.3.3), we get the desired result.

8.4. Remark. Note that Lemma 8.6 of [L3] is no longer necessary; we can simply adopt (8.3.1) as the definition of $\iota(G, H)$. In any case, that lemma follows from our Lemma 8.3.2.

9. Embeddings of tori. Let $F$ be a field, $G$ a connected reductive $F$-group, and $H$ a quasi-split inner form of $G$. Choose a $\Gamma$-isomorphism $\hat{G} \stackrel{\sim}{\rightarrow} \hat{H}$; this determines an inner twisting $\psi: H \rightarrow G$, well-defined up to conjugation by an element of $G$. Let $T$ be a maximal $F$-torus of $H$. We will say that an $F$-embedding $i: T \rightarrow G$ is admissible (relative to $\hat{G} \stackrel{\sim}{\rightarrow} \hat{H}$ ) if it is of the form $\left.\operatorname{Int}(g) \circ \psi\right|_{T}$ for some $g \in G$. Any two admissible $F$-embeddings are stably conjugate (conjugate under $G$ ), but not necessarily $G(F)$-conjugate.

9.1. Consider the unramified situation. Let $F$ be a nonarchimedean local field with valuation ring $\mathrm{o}$, and assume that $G, H, T$ are unramified over $F$ (quasi-split over $F$ and split over an unramified extension of $F$ ). Let $x_{0}$ be a hyperspecial point in the building of $G$ over $F$, and let $K=\operatorname{Stab}_{G(F)}\left(x_{0}\right)$. Then $x_{0}$ determines a smooth o-structure on $G$ (see [Ti]) for which $G\left(\mathfrak{o}_{L}\right)=\operatorname{Stab}_{G(L)}\left(x_{0}\right)$ for any unramified extension $L$ of $F$. Since $T$ is unramified, it extends uniquely to a torus group scheme over $\mathfrak{o}$. For any unramified extension $L$ of $F$ the group $T\left(\mathfrak{o}_{L}\right)$ is the maximal compact subgroup of $T(L)$.

9.1.1. Lemma. Let $i_{1}, i_{2}: T \rightarrow G$ be two admissible F-embeddings, and assume that $i_{1}, i_{2}$ are defined over $\mathrm{o}$. Then $i_{1}, i_{2}$ are conjugate under $K$.

Choose a finite unramified extension $L$ of $F$ that splits $T$ (and hence $G$ ). Since $\left\{g \in G \mid \operatorname{Int}(g) \circ i_{1}=i_{2}\right\}$ is an $F$-torsor under $T$, and since $H^{1}(L, T)$ is trivial, there exists $g \in G(L)$ such that $\operatorname{Int}(g) \circ i_{1}=i_{2}$. We have $i_{1}\left(T\left(\mathfrak{o}_{L}\right)\right) \subset G\left(\mathfrak{o}_{L}\right)$, and therefore $x_{0}$ belongs to the apartment of $i_{1}(T)$. We also have $i_{1}\left(T\left(\mathfrak{o}_{L}\right)\right) \subset g^{-1}$. $G\left(\mathrm{o}_{L}\right) \cdot g$, and therefore $g^{-1} \cdot x_{0}$ also belongs to the apartment of $i_{1}(T)$; from this it follows that $g^{-1} \in i_{1}\left(T\left(\mathfrak{o}_{L}\right)\right) \cdot G\left(\mathfrak{o}_{L}\right)$, and without loss of generality we may assume that $g \in G\left(0_{L}\right)$. Then $\left(g^{-1} \cdot g^{\sigma}\right)_{\sigma \in \operatorname{Gal}(L / F)}$ is a 1-cocycle of $\operatorname{Gal}(L / F)$ in $T(L) \cap G\left(\mathrm{o}_{L}\right)=T\left(\mathrm{o}_{L}\right)$. Since $H^{1}\left(L / F, T\left(\mathrm{o}_{L}\right)\right)=0$, there exists $t \in T\left(\mathrm{o}_{L}\right)$ such that $g^{-1} \cdot g^{\sigma}=t^{-1} \cdot t^{\sigma}$, or, in other words, $g t^{-1} \in G(\mathfrak{D})=K$. This proves the lemma, since $g t^{-1}$ conjugates $i_{1}$ into $i_{2}$.

9.2 Now take $F$ to be a number field, and assume that $G$ has no $E_{8}$ factors, so that $G_{\mathrm{sc}}$ satisfies the Hasse principle. Let $S$ denote the set of all places of $F$. For $v \in S$ we will write $G_{v}$ instead of $G_{F_{v}}$. Suppose that we are given an admissible $F_{v}$-embedding $i(v): T_{v} \rightarrow G_{v}$ for each $v \in S$. When can we find an admissible $F$-embedding $i: T \rightarrow G$ such that $\left(i_{v}\right)_{v \in S}$ and $(i(v))_{v \in S}$ are conjugate under $G\left(\mathrm{~A}_{F}\right)$ ? Here $i_{v}: T_{v} \rightarrow G_{v}$ is the $F_{v}$-embedding obtained from $i$ by extension 
of scalars from $F$ to $F_{v}$. This question is answered in Ch. VII of [L3]. First of all, for almost all $v$ the situation must be unramified. More precisely, let $K$ be a compact open subgroup of $G\left(\mathrm{~A}_{F}^{f}\right)$. Then for all but a finite number of places of $F$, the groups $G_{v}$ and $T_{v}$ are unramified and $K \cap G\left(F_{v}\right)$ is a hyperspecial maximal compact subgroup of $G\left(F_{v}\right)$. For these places we get $\mathfrak{o}_{v}$-structures on $G_{v}, T_{v}$. Any family $(i(v))$ coming from a global $i$ must satisfy the following property:

$$
i(v) \text { is defined over } \mathfrak{o}_{v} \text { for almost all } v \in S .
$$

Now consider a family $(i(v))_{v \in S}$ that satisfies (9.2.1). Langlands constructs an element of $\Re(T / F)^{D}$ whose vanishing is a necessary and sufficient condition for the existence of an admissible $F$-embedding $i: T \rightarrow G$ such that $\left(i_{v}\right)_{v \in S}$ and $(i(v))_{v \in S}$ are conjugate under $G\left(\mathrm{~A}_{F}\right)$. This construction, which involves cocycle calculations, is rather complicated, and the purpose of this section is to give a simpler construction. We will again get an obstruction lying in $\Re(T / F)^{D}$, and undoubtedly the two obstructions are the same, at least up to a sign, but we will not stop to check this. It would not be unreasonable simply to reformulate the global hypothesis on p. 149 of [L3] in terms of the new obstruction.

Now we will see how to get our obstruction. Let $\mathscr{F}_{0}$ be the set of pairs $(i, g)$ satisfying

$$
i \text { is an admissible } \bar{F} \text {-embedding } T_{\bar{F}} \rightarrow G_{\bar{F}} \text {, }
$$

$$
\begin{aligned}
& \left.g=\left(g_{w}\right)_{w \in \bar{S}} \in G_{\mathrm{sc}}\left(\mathrm{A}_{\bar{F}}\right) \text { (here } \bar{S} \text { is the set of places of } \bar{F}\right), \\
& i_{w}=\operatorname{Int}\left(g_{w}\right) \circ i(v)_{w} \text { for every } v \in S \text { and every } w \in \bar{S} \\
& \text { lying over } v \text {. }
\end{aligned}
$$

In (9.2.4) the subscript $w$ on $i$ and $i(v)$ denotes extension of scalars to $\bar{F}_{w}$. We have three actions on $\mathscr{F}_{0}$ :

(i) $\Gamma$ acts on $\mathscr{F}_{0}$ by $(i, g)^{\sigma}=\left(i^{\sigma}, g^{\sigma}\right)(\sigma \in \Gamma)$,

(ii) $G_{\mathrm{sc}}(\bar{F})$ acts on $\mathscr{F}_{0}$ by $x \cdot(i, g)=(\operatorname{Int}(x) \circ i, x g)\left(x \in G_{\mathrm{sc}}(\bar{F})\right)$,

(iii) $T_{\mathrm{sc}}\left(\mathrm{A}_{\bar{F}}\right)$ acts on $\mathscr{F}_{0}$ by $(i, g) \cdot t=\left(i, i\left(t^{-1}\right) \cdot g\right)\left(t \in T_{\mathrm{sc}}\left(\mathrm{A}_{\bar{F}}\right)\right)$.

We have the following compatibilities:

(a) $[x \cdot(i, g)]^{\sigma}=x^{\sigma} \cdot(i, g)^{\sigma}$ for $x \in G_{\mathrm{sc}}(\bar{F}), \sigma \in \Gamma$,

(b) $[(i, g) \cdot t]^{\sigma}=(i, g)^{\sigma} \cdot t^{\sigma}$ for $t \in T_{\mathrm{sc}}\left(\mathrm{A}_{\bar{F}}\right), \sigma \in \Gamma$,

(c) the actions of $G_{\mathrm{sc}}(\bar{F})$ and $T_{\mathrm{sc}}\left(\mathrm{A}_{\bar{F}}\right)$ commute.

Let $\mathscr{F}$ denote the quotient of $\mathscr{F}_{0}$ by $G_{\text {sc }}(\bar{F})$. We have induced actions of $\Gamma$ and $T_{\mathrm{sc}}\left(\mathrm{A}_{\bar{F}}\right)$ on $\mathscr{F}$, and it is not hard to check that $\mathscr{F}$ is an $F$-torsor under $T_{\mathrm{sc}}\left(\mathrm{A}_{\bar{F}}\right) / T_{\mathrm{sc}}(\bar{F})$.

9.2.5. LEMMA. The following are equivalent:

(i) There exists an admissible $F$-embedding $i: T \rightarrow G$ such that $\left(i_{v}\right),(i(v))$ are 
conjugate under $G_{\mathrm{sc}}\left(\mathrm{A}_{F}\right)$.

(ii) The set $\mathscr{F}_{0}^{\mathrm{rc}}$ is nonempty.

(iii) The torsor $\mathscr{F}$ is trivial.

It is obvious that (i) and (ii) are equivalent and that (ii) implies (iii). We will now check that (iii) implies (ii). Assume that $\mathscr{F}$ is trivial. The $\mathscr{F}^{\Gamma}$ is nonempty. Choose an element of $\mathscr{F} \Gamma$, and let $\mathscr{F}_{1}$ be the fiber of $\mathscr{F}_{0} \rightarrow \mathscr{F}$ over that element. Then $\mathscr{F}_{1}$ is an $F$-torsor under $G_{\text {sc }}(\bar{F})$. To finish the proof we must show that $\mathscr{F}_{1}$ is trivial. Choose $(i, g) \in \mathscr{F}_{1}$. The associated 1-cocycle $\left(x_{\sigma}\right)$ of $\Gamma$ in $G_{\mathrm{sc}}(\bar{F})$ is determined by the equation

$$
(i, g)^{\sigma}=x_{\sigma} \cdot(i, g)
$$

In particular, we have $x_{\sigma}=g^{\sigma} \cdot g^{-1}$ with $g \in G_{\text {sc }}\left(A_{\bar{F}}\right)$. Hence $\left(x_{\sigma}\right)$ is trivial in $H^{1}\left(F, G_{\text {sc }}\left(\mathrm{A}_{\bar{F}}\right)\right)$. Since we are assuming that $G$ has no $E_{8}$ factors, the Hasse principle holds for $G_{\text {sc }}$, and we see that $\mathscr{F}_{1}$ is trivial.

9.3. We retain the notation of 9.2. Associated to $\mathscr{F}$ is a cohomology class $c \in H^{1}\left(F, T_{\mathrm{sc}}\left(\mathrm{A}_{\bar{F}}\right) / T_{\mathrm{sc}}(\bar{F})\right)$. By Tate-Nakayama duality (3.4.2.2) and Lemma 2.2 this cohomology group is canonically isomorphic to $\Re\left(T_{\mathrm{sc}} / F\right)^{D}$. If $G=G_{\mathrm{sc}}$, then the element $c$, viewed in $\Re\left(T_{\mathrm{sc}} / F\right)^{D}$, is the desired obstruction. In the general case we have $\mathscr{A}(T / K) \subset \Re\left(T_{\text {sc }} / F\right)$ and hence a canonical surjection

$$
\Re\left(T_{\mathrm{sc}} / F\right)^{D} \rightarrow \Re(T / F)^{D} .
$$

The image of $c$ in $\AA(T / F)^{D}$ is the obstruction we want, but before we can prove this we need to establish some preliminary results.

9.4. We retain the notation of 9.2 and 9.3. It is easy to find a 1-cocycle of $\Gamma$ in $T_{\mathrm{sc}}\left(\mathrm{A}_{\bar{F}}\right) / T_{\mathrm{sc}}(\bar{F})$ that represents the cohomology class $c$. We choose $(i, g) \in \mathscr{F}$ and extend $i: T \rightarrow G$ to an $\bar{F}$-isomorphism $i: H \stackrel{\sim}{\rightarrow} G$ (recall that $H$ is a quasi-split inner form of $G$ ). Then $i$ is an inner twisting (up to conjugation by $G$ it is the same as $\psi: H \rightarrow G)$; therefore we can choose, for $\sigma \in \Gamma$, an element $h_{\sigma} \in H_{\mathrm{sc}}(\bar{F})$ such that $i^{-1} \circ i^{\sigma}=\operatorname{Int}\left(h_{\sigma}\right)$. Then $\left(\operatorname{Int}\left(h_{\sigma}\right)\right)_{\sigma \in \Gamma}$ represents the cohomology class in $H^{1}\left(F, H_{\text {ad }}\right)$ associated to the inner twisting $\psi: H \rightarrow G$. An easy calculation shows that $c$ can be represented by the 1-cocycle $\left(t_{\sigma}\right)$, where $t_{\sigma}$ denotes the image in $T_{\mathrm{sc}}\left(\mathrm{A}_{\bar{F}}\right) / T_{\mathrm{sc}}(\bar{F})$ of the element $i^{-1}\left(g \cdot g^{-\sigma}\right) \cdot h_{\sigma}$ of $T_{\mathrm{sc}}\left(\mathrm{A}_{\bar{F}}\right)$.

9.4.1. Remark. Let $c^{\prime}$ denote the image of $c$ under the homomorphism

$$
H^{1}\left(F, T_{\mathrm{sc}}\left(\mathrm{A}_{\bar{F}}\right) / T_{\mathrm{sc}}(\bar{F})\right) \rightarrow H^{2}\left(F, T_{\mathrm{sc}}\right)
$$

induced by the exact sequence

$$
1 \rightarrow T_{\mathrm{sc}}(\bar{F}) \rightarrow T_{\mathrm{sc}}\left(\mathrm{A}_{\bar{F}}\right) \rightarrow T_{\mathrm{sc}}\left(\mathrm{A}_{\bar{F}}\right) / T_{\mathrm{sc}}(\bar{F}) \rightarrow 1
$$

By direct calculation we see that $c^{\prime}$ is equal to the image of $\left(\operatorname{Int}\left(h_{\sigma}\right)\right)$ under

$$
H^{1}\left(F, H_{\mathrm{ad}}\right) \rightarrow H^{2}\left(F, Z\left(H_{\mathrm{sc}}\right)\right) \rightarrow H^{2}\left(F, T_{\mathrm{sc}}\right) .
$$


9.4.2. We will need to know the effect on $c$ of a change in the family $(i(v))$. Use the family $i(v)$ to build a homomorphism $i(\mathrm{~A}): T\left(\mathrm{~A}_{\bar{F}}\right) \rightarrow G\left(\mathrm{~A}_{\bar{F}}\right)$. Then consider a 1-cocycle $\left(d_{\sigma}\right)$ of $\Gamma$ in $T\left(\mathrm{~A}_{\bar{F}}\right)$ whose image under

$$
H^{1}\left(F, T\left(\mathrm{~A}_{\bar{F}}\right)\right) \rightarrow H^{1}\left(F, G\left(\mathrm{~A}_{\bar{F}}\right)\right)
$$

is trivial. Then there exists $x=\left(x_{w}\right)_{w \in \bar{S}} \in G\left(\mathrm{~A}_{\bar{F}}\right)$ such that $i(\mathrm{~A})\left(d_{\sigma}\right)=x^{-1} \cdot x^{\sigma}$ for all $\sigma \in \Gamma$. Let $v \in S$. We get an admissible $F_{v}$-embedding $j(v): T_{v} \rightarrow G_{v}$ by putting $j(v)=\operatorname{Int}\left(x_{w}\right) \circ i(v)$ for $w$ lying over $v$ (this is independent of $w$ ). The $G\left(\mathrm{~A}_{F}\right)$-conjugacy class of $(j(v))_{v \in S}$ is independent of the choice of $x$, and the map $\left(d_{\sigma}\right) \mapsto(j(v))$ induces a bijection from

$$
\operatorname{ker}\left[H^{1}\left(F, T\left(\mathrm{~A}_{\bar{F}}\right)\right) \rightarrow H^{1}\left(F, G\left(\mathrm{~A}_{\bar{F}}\right)\right)\right]
$$

to the set of $G\left(\mathrm{~A}_{F}\right)$-conjugacy classes of families $(j(v))_{v \in S}$ satisfying (9.2.1). There is a bijection between the set of admissible embeddings of $T$ in $G$ and the set of admissible embeddings of $T_{\mathrm{sc}}$ in $G_{\mathrm{sc}}$; thus, applying the discussion above to $T_{\text {sc }}, G_{\text {sc }}$, we also obtain a bijection from

$$
\operatorname{ker}\left[H^{1}\left(F, T_{\mathrm{sc}}\left(\mathrm{A}_{\bar{F}}\right)\right) \rightarrow H^{1}\left(F, G_{\mathrm{sc}}\left(\mathrm{A}_{\bar{F}}\right)\right)\right]
$$

to the set of $G_{\mathrm{sc}}\left(\mathrm{A}_{\bar{F}}\right)$-conjugacy classes of families $(j(v))_{v \in S}$ satisfying (9.2.1). Let $d$ be an element of the group (9.4.2.2) and let $(j(v))$ be the corresponding family. Let $c_{\text {new }}$ be the element of $H^{1}\left(F, T_{\text {sc }}\left(\mathrm{A}_{\bar{F}}\right) / T_{\text {sc }}(\bar{F})\right)$ associated to $(j(v))$ in the same way that $c$ is associated to $(i(v))$. Using the method given above to find 1-cocycles representing $c$ and $c_{\text {new }}$, we see that $c_{\text {new }}$ is the sum of $c$ and the image of $d$ under

$$
H^{1}\left(F, T_{\mathrm{sc}}\left(\mathrm{A}_{\bar{F}}\right)\right) \rightarrow H^{1}\left(F, T_{\mathrm{sc}}\left(\mathrm{A}_{\bar{F}}\right) / T_{\mathrm{sc}}(\bar{F})\right) .
$$

9.4.3. From 9.4.2 we see that in order to prove that the image of $c$ in $\Re(T / F)^{D}$ is the obstruction we want, it is enough to prove the equality of the following two subsets of $H^{1}\left(F, T_{\mathrm{sc}}\left(\mathrm{A}_{\bar{F}}\right) / T_{\mathrm{sc}}(\bar{F})\right)=\Re\left(T_{\mathrm{sc}} / F\right)^{D}$ :

(a) the kernel of the homomorphism (9.3.1),

(b) the image in $H^{1}\left(F, T_{\mathrm{sc}}\left(\mathrm{A}_{\bar{F}}\right) / T_{\mathrm{sc}}(\bar{F})\right)$ of the subset $A$ of $H^{1}\left(F, T_{\mathrm{sc}}\left(\mathrm{A}_{\bar{F}}\right)\right)$ consisting of elements that have trivial image in both $H^{1}\left(F, T\left(A_{\bar{F}}\right)\right)$ and $H^{1}\left(F, G_{\text {sc }}\left(\mathrm{A}_{\bar{F}}\right)\right)$.

The first step in proving that the sets (a) and (b) are equal is to note that neither set changes when $G$ is replaced by a $z$-extension $G^{\prime}$ of $G$ (see 7.8). Thus, without loss of generality, we may assume that $G_{\text {der }}$ is simply connected. Let $D=G / G_{\mathrm{der}}$.

The next step is to show that the set (b) is equal to the image in $H^{1}\left(F, T_{\text {sc }}\left(\mathrm{A}_{\bar{F}}\right) / T_{\text {sc }}(\bar{F})\right)$ of $B$, where

$$
B=\operatorname{ker}\left[H^{1}\left(F, T_{\mathrm{sc}}\left(\mathrm{A}_{\bar{F}}\right)\right) \rightarrow H^{1}\left(F, T\left(\mathrm{~A}_{\bar{F}}\right)\right)\right]
$$

Since $A \subset B$, it is obvious that this set contains the set (b). To check the reversed 
inclusion one uses the long exact cohomology sequence for

$$
1 \rightarrow T_{\mathrm{sc}} \rightarrow T \rightarrow D \rightarrow 1
$$

and the fact that $D(F)$ is dense in $D\left(F \otimes_{\mathrm{Q}} \mathrm{R}\right)$.

The last step is to show that (a) is equal to the image of $B$. Consider the diagram

$$
\begin{aligned}
& H^{1}\left(F, T_{\mathrm{sc}}\left(\mathrm{A}_{\bar{F}}\right)\right) \stackrel{\alpha}{\longrightarrow} H^{1}\left(F, T_{\mathrm{sc}}\left(\mathrm{A}_{\bar{F}}\right) / T_{\mathrm{sc}}(\bar{F})\right) \\
& \quad \beta \downarrow \\
& H^{1}\left(F, T\left(\mathrm{~A}_{\bar{F}}\right)\right) .
\end{aligned}
$$

Taking duals, we get the diagram

$$
\begin{array}{r}
\Re\left(T_{\mathrm{sc}} / F\right) \stackrel{\alpha^{D}}{\longrightarrow} \prod_{v} \Re\left(T_{\mathrm{sc}} / F_{v}\right) \\
\uparrow \beta^{D} \\
\prod_{v} \pi_{0}\left(\hat{T}^{\Gamma_{v}}\right),
\end{array}
$$

where $\Gamma_{v}$ is the absolute Galois group of $F_{v}$. By definition, $\Re(T / F)$ is the inverse image under $\alpha^{D}$ of $\operatorname{im}\left(\beta^{D}\right)$, or, in other words,

$$
\Re(T / F)=\operatorname{ker}\left[\Re\left(T_{\mathrm{sc}} / F\right) \rightarrow \operatorname{cok}\left(\beta^{D}\right)\right] .
$$

Therefore $\Re(T / F)^{D}$ is equal to

$$
\operatorname{cok}\left[\operatorname{ker} \beta \rightarrow \Re\left(T_{\mathrm{sc}} / F\right)^{D}\right]
$$

which shows that the image of $B=\operatorname{ker} \beta$ in $H^{1}\left(F, T_{\mathrm{sc}}\left(\mathrm{A}_{\bar{F}}\right) / T_{\mathrm{sc}}(\bar{F})\right)$ is equal to the kernel of

$$
\Re\left(T_{\mathrm{sc}} / F\right)^{\mathcal{D}} \rightarrow \Re(T / F)^{D},
$$

which is the definition of the subgroup (a).

9.5. Let $G, H, T$ be as in the beginning of the section. We will say that $T$ transfers to $G$ (with respect to $\hat{G} \tilde{\rightarrow} \hat{H}$ ) if there exists an admissible $F$-embedding $T \rightarrow G$. Now consider the case in which $F$ is a global field and $T_{v}$ transfers to $G_{v}$ for every place $v$ of $F$. As before, we assume that $G$ has no $E_{8}$ factors. Langlands [L3] constructs an obstruction which vanishes if and only if $T$ transfers to $G$. It is easy to understand this in terms of the results of this section. Since $T$ transfers to $G$ locally, we can choose a family $(i(v))_{v \in S}$ as in 9.2 . We can assume that the family satisfies (9.2.1). This gives us an element $c \in H^{1}\left(F, T_{\mathrm{sc}}\left(\mathrm{A}_{\bar{F}}\right) / T_{\mathrm{sc}}(\bar{F})\right)$. It is obvious that $T$ transfers to $G$ if and only if there exists a family $(j(v))_{v \in S}$ for 
which $c+e$ vanishes, where $e$ is the image in $H^{1}\left(F, T_{\mathrm{sc}}\left(\mathrm{A}_{\bar{F}}\right) / T_{\mathrm{sc}}(\bar{F})\right)$ of the element $d \in H^{1}\left(F, T_{\mathrm{sc}}\left(\mathrm{A}_{\bar{F}}\right)\right)$ corresponding to $(j(v))_{v \in S}$ (see 9.4.2). In other words, $T$ transfers to $G$ if and only if $c$ belongs to the image in $H^{1}\left(F, T_{\mathrm{sc}}\left(\mathrm{A}_{\bar{F}}\right) / T_{\mathrm{sc}}(\bar{F})\right)$ of the group (9.4.2.2). It is easy to see that this image is the same as the image of the whole group $H^{1}\left(F, T_{\mathrm{sc}}\left(\mathrm{A}_{\bar{F}}\right)\right.$ ) (use the fact that $H^{1}\left(F, T_{\mathrm{sc}}\right)$ maps onto $\prod_{v \in S_{\infty}} H^{1}\left(F_{v}, T_{\mathrm{sc}}\right)$, where $S_{\infty}$ is the set of infinite places of $F)$. Using the long exact cohomology sequence for

$$
1 \rightarrow T_{\mathrm{sc}}(\bar{F}) \rightarrow T_{\mathrm{sc}}\left(\mathrm{A}_{\bar{F}}\right) \rightarrow T_{\mathrm{sc}}\left(\mathrm{A}_{\bar{F}}\right) / T_{\mathrm{sc}}(\bar{F}) \rightarrow 1,
$$

we see that $T$ transfers to $G$ if and only if the image $c^{\prime}$ of $c$ in $H^{2}\left(F, T_{\text {sc }}\right)$ is trivial. We have seen in Remark 9.4.1 that $c^{\prime}$ can be calculated directly from the class in $H^{1}\left(F, H_{\text {ad }}\right)$ that corresponds to the inner twisting $\psi: H \rightarrow G$. In fact, this alternative definition of $c^{\prime}$ can also be used directly to show that $T$ transfers to $G$ if and only if $c^{\prime}$ is trivial.

10. Admissible homomorphisms. In this section $F$ is a local or global field and $W_{F}$ is the Weil group of $\bar{F} / F$. For a connected reductive group $G$ over $F$ we write ${ }^{L} G$ for the Weil form $W_{F} \ltimes \hat{G}$ of the $L$-group of $G$. In the global case, given a place $v$ of $F$, we write $G_{v}$ for $G_{F_{v}}$. We consider continuous splittings $\varphi: W_{F} \rightarrow{ }^{L} G$ of ${ }^{L} G \rightarrow W_{F}$. In the global case, given a place of $v$ of $F$, we have a homomorphism $\theta_{v}: W_{F_{v}} \rightarrow W_{F}$, canonical up to conjugation by an element of $W_{F}$ (see [T3]), and $\theta_{v}$ induces a homomorphism ${ }^{L}\left(G_{v}\right) \rightarrow^{L} G$. There is a unique homomorphism $\varphi_{v}: W_{F_{\mathrm{v}}} \rightarrow^{L}\left(G_{v}\right)$ such that

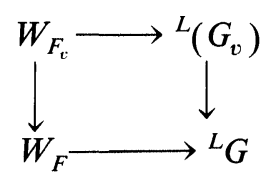

commutes. As in (2.1) of [T3] we set that $\varphi \mapsto \varphi_{v}$ induces a well-defined map from the set of homomorphisms $\varphi: W_{F} \rightarrow{ }^{L} G$ as above, taken modulo conjugation by $\hat{G}$, to the analogous local set.

We say that $\varphi: W_{F} \rightarrow{ }^{L} G$ is admissible if

(i) $\varphi(w)$ is semisimple in ${ }^{L} G$ for all $w \in W_{F}$ (an element of ${ }^{L} G$ is said to be semisimple if its projection in the algebraic group $\operatorname{Gal}(K / F) \ltimes \hat{G}$ is semisimple for every finite Galois extension $K / F$ that splits $G$ ),

(ii) $\varphi$ is locally relevant: in the local case this means that if $\varphi$ factors through a Levi subgroup of a parabolic subgroup of ${ }^{L} G$, then the parabolic subgroup is relevant; in the global case this means that $\varphi_{v}$ is relevant for every place $v$ of $F$.

It is obvious that $\varphi_{v}$ is admissible if $\varphi$ is admissible.

10.1. Let $\varphi: W_{F} \rightarrow^{L} G$ be an admissible homomorphism. We will write $C_{\varphi}$ for the centralizer of $\varphi\left(W_{F}\right)$ in $\hat{G}$.

10.1.1. LemMA. The group $C_{\varphi}$ is reductive (not necessarily connected).

Let $W_{F}^{1}$ denote the kernel of \|\|$: W_{F} \rightarrow \mathrm{R}_{+}$, and let $A$ (resp. $B$ ) denote the 
Zariski closure of $p\left(\varphi\left(W_{F}\right)\right)$ (resp. $\left.p\left(\varphi\left(W_{F}^{1}\right)\right)\right)$ in $\operatorname{Aut}(\hat{G})$, where $p:{ }^{L} G \rightarrow \operatorname{Aut}(\hat{G})$ is the homomorphism determined by the action of ${ }^{L} G$ on $\hat{G}$ by conjugation. Since $W_{F}^{1}$ is compact (see [T3]), $B$ is reductive. There is a continuous homomorphism $\mathrm{R}_{+} \rightarrow A / B$, whose image is Zariski dense in $A / B$ and consists of semisimple elements (by part (i) of the definition of admissible homomorphism). Therefore $A / B$ is a torus, which implies that $A$ is reductive. The group $C_{\varphi}$ is equal to $\hat{G}^{A}$, the group of fixed points of $A$ in $\hat{G}$. The fact that $C_{\varphi}$ is reductive is a consequence of the following lemma.

10.1.2. Lemma. Let $k$ be an algebraically closed field of characteristic $0, G a$ connected reductive group over $k$, and $A$ a reductive subgroup of $\operatorname{Aut}_{k}(G)$. Then the group $G^{A}$ of fixed points of $A$ in $G$ is reductive.

The first step is to note that if $k^{\prime}$ is an algebraically closed extension field of $k$, then $\left(G^{A}\right)\left(k^{\prime}\right)=G\left(k^{\prime}\right)^{A\left(k^{\prime}\right)}$. Using this, we reduce to the case in which $k$ is finitely generated over $\mathrm{Q}$; using it again, we reduce to the case that $k=\mathrm{C}$.

Now we assume that $k=\mathrm{C}$. There exists a real structure on $A$ for which $A(\mathrm{R})$ is compact and Zariski dense in $A(\mathrm{C})$ (see [H]). We have $G^{A}=G^{K}$, where $K=A(\mathrm{R})$.

It is now sufficient to prove the following statement: if $K$ is a compact subgroup of $\operatorname{Aut}(G)$ then $G^{K}$ is reductive. Choose a real structure on $G$ for which $G(\mathrm{R})$ is compact. For this real structure the group $\operatorname{Aut}(G)(\mathrm{R})$ is a maximal compact subgroup of $\operatorname{Aut}(G)$. Replacing $K$ by a conjugate under $\operatorname{Aut}(G)$, we may assume $K$ is contained in $\operatorname{Aut}(G)(\mathrm{R})$. Then $G^{K}$ is defined over $\mathrm{R}$. Furthermore, $G^{K}(\mathrm{R})$ is compact, for it is a closed subgroup of $G(\mathrm{R})$. Therefore $G^{K}$ is reductive.

10.2. Let $\varphi: W_{F} \rightarrow^{L} G$ be an admissible homomorphism. We define a group $D_{\varphi}$ containing $C_{\varphi}$ (see 10.1$)$ and $Z(\hat{G})$ by putting

$$
D_{\varphi}=\left\{s \in \hat{G} \mid s \cdot \varphi(w) \cdot s^{-1} \cdot \varphi(w)^{-1} \in Z(\hat{G}) \text { for all } w \in W_{F}\right\} .
$$

The groups $C_{\varphi}^{0}, D_{\varphi}^{0}$ have the same image in the adjoint group of $\hat{G}$, as one sees by considering Lie algebras, and therefore

$$
C_{\varphi}^{0} \cdot Z(\hat{G})=D_{\varphi}^{0} \cdot Z(\hat{G}) .
$$

It follows from Lemma 10.1.1 that $D_{\varphi}$ is reductive.

For $s \in D_{\varphi}$ and $w \in W_{F}$ define an element $z_{w} \in Z(\hat{G})$ by

$$
z_{w}=s \cdot \varphi(w) \cdot s^{-1} \cdot \varphi(w)^{-1} .
$$

Then $\left(z_{w}\right)$ is a continuous 1-cocycle of $W_{F}$ in $Z(\hat{G})$, and $s \mapsto\left(z_{w}\right)$ is a homomorphism from $D_{\varphi}$ to the group of continuous 1-cocycles. Let $H^{1}\left(W_{F}\right.$, $Z(\hat{G}))$ denote the group of continuous 1-cocycles modulo 1-coboundaries. We get a homomorphism

$$
D_{\varphi} \rightarrow H^{1}\left(W_{F}, Z(\hat{G})\right) \text {. }
$$


Let $S_{\varphi}$ denote the subgroup of $D_{\varphi}$ consisting of elements $s$ whose image in $H^{1}\left(W_{F}, Z(\hat{G})\right)$ is

(a) trivial if $F$ is local,

(b) locally trivial if $F$ is global, or, in other words, an element of $\operatorname{ker}^{1}\left(W_{F}, Z(\hat{G})\right)$, the kernel of $H^{1}\left(W_{F}, Z(\hat{G})\right) \rightarrow \Pi_{v} H^{1}\left(W_{F_{v}}, Z(\hat{G})\right)$.

Finally, we define a group $\mathfrak{S}_{\varphi}$ by putting

$$
\Im_{\varphi}=S_{\varphi} / S_{\varphi}^{0} \cdot Z(\hat{G})=\pi_{0}\left(S_{\varphi} / Z(\hat{G})\right)
$$

Using (10.2.1), we see that

$$
C_{\varphi}^{0} \cdot Z(\hat{G})=S_{\varphi}^{0} \cdot Z(\hat{G}) .
$$

In the local case we have $\mathfrak{S}_{\varphi}=C_{\varphi} / C_{\varphi}^{0} \cdot Z(\hat{G})^{\Gamma}$, which agrees with the usual definition. In the global case we have an exact sequence

$$
1 \rightarrow C_{\varphi} / C_{\varphi}^{0} \cdot Z(\hat{G})^{\Gamma} \rightarrow \Im_{\varphi} \rightarrow \operatorname{ker}^{1}\left(W_{F}, Z(\hat{G})\right)
$$

and the definition of $\widetilde{S}_{\varphi}$ is the obvious generalization of the definition in [L-L].

An admissible homomorphism $\varphi: W_{F} \rightarrow^{L} G$ is said to be tempered if the image of $\varphi\left(W_{F}\right)$ in $\operatorname{Gal}(K / F) \ltimes \hat{G}$ is relatively compact in $\operatorname{Gal}(K / F) \ltimes \hat{G}$ for every finite Galois extension $K / F$ that splits $G$. Let $\varphi: W_{F} \rightarrow^{L} G$ be a tempered admissible homomorphism. In the local case, Langlands [L3] conjectures that the group $\mathfrak{S}_{\varphi}$ controls the $L$-packet $\Pi(\varphi)$ (see [Sh] for the archimedean case). In the global case, Labesse and Langlands [L-L] conjecture that the group $\mathfrak{S}_{\varphi}$ controls the automorphic representations in the $L$-packet $\Pi(\varphi)$.

10.3. Let $\varphi: W_{F} \rightarrow^{L} G$ be an admissible homomorphism. We will say that $\varphi$ is elliptic if $\varphi$ factors through no proper Levi subgroup of ${ }^{L} G$. If $\varphi$ is elliptic and tempered, then one expects that $\Pi(\varphi)$ consists of cuspidal representations in the local case, and one expects that the automorphic elements of $\Pi(\varphi)$ are cuspidal in the global case.

10.3.1. LEMma. The following are equivalent:

(i) $\varphi$ is elliptic.

(ii) $C_{\varphi}^{0} \subset Z(\hat{G})$,

(iii) $S_{\varphi}^{0} \subset Z(\hat{G})$.

The equivalence of (ii) and (iii) is obvious from (10.2.4). The equivalence of (i) and (ii) follows from Lemma 3.5 of [B] and the fact that $C_{\varphi}$ is reductive (Lemma 10.1.1), from which it follows that $C_{\varphi}^{0} \subset Z(\hat{G})$ if and only if every torus in $\hat{G}$ that commutes with $\varphi$ is contained in $Z(\hat{G})$ (note that the centralizer in ${ }^{L} G$ of a torus that commutes with $\varphi$ must project onto $W_{F}$, since it contains $\varphi\left(W_{F}\right)$ ).

10.4. Let $\varphi_{1}, \varphi_{2}: W_{F} \rightarrow{ }^{L} G$ be admissible homomorphisms. We say that $\varphi_{1}, \varphi_{2}$ are equivalent if there exists $g \in \hat{G}$ such that $\varphi_{1}=\left(\operatorname{Int}(g) \circ \varphi_{2}\right) \cdot z$ for some continuous 1-cocycle $z=\left(z_{w}\right)$ of $W_{F}$ in $Z(\hat{G})$ whose class in $H^{1}\left(W_{F}, Z(\hat{G})\right)$ is

(a) trivial if $F$ is local,

(b) locally trivial if $F$ is global. 
We will use $[\varphi]$ to denote the equivalence class of $\varphi$. It is not hard to see that the properties of being tempered or elliptic depend only on the equivalence class of an admissible homomorphism. Note that $S_{\varphi}$ can be thought of as the group of self-equivalences of $\varphi$. If $\varphi_{1}, \varphi_{2}$ differ by a 1-cocycle of $W_{F}$ in $Z(\hat{G})$, then $S_{\varphi_{1}}=S_{\varphi_{2}}$. If $\varphi_{1}, \varphi_{2}$ are equivalent, then by choosing $g \in \hat{G}$ as above we get $\operatorname{Int}(g): S_{\varphi_{2}} \tilde{\rightarrow} S_{\varphi_{1}}$, well-defined up to inner automorphisms of $S_{\varphi_{1}}$. The same remarks apply to $\widetilde{\Im}_{\varphi_{1}}, \widetilde{\subseteq}_{\varphi_{2}}$.

11. Admissible homomorphisms for endoscopic groups. Let $F$ be a number field and let $G$ be a connected reductive group over $F$. In this section we will study the relationship between elliptic admissible homomorphisms for $G$ and elliptic admissible homomorphisms for the elliptic endoscopic groups of $G$. We will use these results when we discuss the stabilization of the trace formula for $G$.

11.1. Let $(H, s, \eta)$ be an endoscopic triple for $G$. Then $Z(\hat{G})$ is a $\Gamma$-submodule of $Z(\hat{H})$ (see 7.4), and we can use $Z(\hat{G})$ to define a new equivalence relation on the set of admissible homomorphisms $\psi: W_{F} \rightarrow{ }^{L} H$. We say that two admissible embeddings $\psi_{1}, \psi_{2}$ for $H$ are $Z(\hat{G})$-equivalent if there exists $h \in \hat{H}$ such that $\psi_{1}=\left(\operatorname{Int}(h) \circ \psi_{2}\right) \cdot z$ for some continuous 1-cocycle $z=\left(z_{w}\right)$ of $W_{F}$ in $Z(\hat{G})$ whose class in $H^{1}\left(W_{F}, Z(\hat{G})\right)$ is locally trivial. It is obvious that $Z(\hat{G})-$ equivalence implies equivalence. We will use $[[\psi]]$ to denote the $Z(\hat{G})$ equivalence class of $\psi$.

It is easy to understand why we have introduced $Z(\hat{G})$-equivalence. Consider an $L$-homomorphism $\eta^{\prime}:{ }^{L} H \rightarrow{ }^{L} G$ extending $\eta: \hat{H} \rightarrow \hat{G}$. Then we have a map $\psi \mapsto \eta^{\prime} \circ \psi$ from the set of admissible homomorphisms for $H$ to the set of admissible homomorphisms for $G$. If $\psi_{1}, \psi_{2}$ are $Z(\hat{G})$-equivalent, then $\eta^{\prime} \circ \psi_{1}$, $\eta^{\prime} \circ \psi_{2}$ are equivalent. Thus $Z(\hat{G})$-equivalence is suitable for the study of the relationship between admissible homomorphisms for $H$ and admissible homomorphisms for $G$.

Let $\psi: W_{F} \rightarrow{ }^{L} H$ be an admissible homomorphism. We denote by ${ }_{G} S_{\psi}$ the group of self-equivalences of $\psi$ for $Z(\hat{G})$-equivalence; in other words, ${ }_{G} S_{\psi}$ is the subgroup of $\hat{H}$ consisting of all $h \in \hat{H}$ such that $\operatorname{Int}(h) \circ \psi$ and $\psi$ differ by a continuous 1-cocycle of $W_{F}$ in $Z(\hat{G})$ whose class in $H^{1}\left(W_{F}, Z(\hat{G})\right)$ is locally trivial. It is obvious that ${ }_{G} S_{\psi}$ contains $Z(\hat{G})$. We define ${ }_{G} \Im_{\psi}$ in analogy with $\widetilde{\Im}_{\psi}$ :

$$
{ }_{G} \widetilde{S}_{\psi}={ }_{G} S_{\psi} /{ }_{G} S_{\psi}^{0} \cdot Z(\hat{G})=\pi_{0}\left({ }_{G} S_{\psi} / Z(\hat{G})\right) \text {. }
$$

11.2. Let $(H, s, \eta)$ be an elliptic endoscopic triple for $G$, and let $\psi: W_{F} \rightarrow{ }^{L} H$ be an elliptic admissible homomorphism for $H$. Recall that we use $\tau_{1}(G)$ to denote the relative Tamagawa number of $G$ (see Section 5).

11.2.1. Proposition. The number of $Z(\hat{G})$-equivalence classes contained in the equivalence class of $\psi$ is equal to

$$
\tau_{1}(G) \cdot \tau_{1}(H)^{-1} \cdot\left|{ }_{G} \Im_{\psi}\right| \cdot\left|\Im_{\psi}\right|^{-1} .
$$


Before proving this proposition, we need to prove a lemma. Let $\operatorname{ker}^{1}\left(W_{F}\right.$, $Z(\hat{H}))$ denote the kernel of $H^{1}\left(W_{F}, Z(\hat{H})\right) \rightarrow \prod_{v} H^{1}\left(W_{F_{v}}, Z(\hat{H})\right)$.

11.2.2. LeMMA. The inflation homomorphism $\operatorname{ker}^{1}(F, Z(\hat{H})) \rightarrow \operatorname{ker}^{1}\left(W_{F}, Z(\hat{H})\right)$ is an isomorphism.

The injectivity of the map is obvious. We will now verify the surjectivity. Let $\alpha$ be a continuous 1-cocycle of $W_{F}$ in $Z(\hat{H})$ whose class is locally trivial. Let $K / F$ be a finite Galois extension that splits $H$. Then $W_{K}$ acts trivially on $Z(\hat{H})$, and therefore the restriction of $\alpha$ to $W_{K}$ is a continuous homomorphism $\beta: W_{K}$ $\rightarrow Z(\hat{H})$. Using class field theory, we view $\beta$ as a continuous homomorphism $\mathrm{A}_{K}^{\times} / K^{\times} \rightarrow Z(\hat{H})$ whose restriction to $K_{w}^{\times}$is trivial for every place $w$ of $K$. Since the sum of the groups $K_{w}^{\times}$is dense in $A_{K}^{\times}$, we see that $\beta$ is trivial. Therefore $\alpha$ is the inflation of a 1-cocycle $\alpha^{\prime}$ of $\operatorname{Gal}(K / F)$ in $Z(\hat{H})$. The class of $\alpha^{\prime}$ in $H^{1}(F, Z(\hat{H}))$ is locally trivial and maps to $\alpha$.

11.2.3. Now we prove Proposition 11.2.1. Our problem is to count the number of $Z(\hat{G})$-equivalence classes $\left[\left[\psi^{\prime}\right]\right]$ contained in the equivalence class $[\psi]$. It is enough to consider $\psi^{\prime}$ of the form $\psi \cdot z$ where $z$ is a continuous 1-cocycle of $W_{F}$ in $Z(\hat{H})$ whose class $\alpha \in H^{1}\left(W_{F}, Z(\hat{H})\right)$ belongs to $\operatorname{ker}^{1}\left(W_{F}, Z(\hat{H})\right)$. From now on we use Lemma 11.2.2 to replace $\operatorname{ker}^{1}\left(W_{F}, Z(\hat{H})\right)$ by $\operatorname{ker}^{1}(F, Z(\hat{H}))$. It is easy to see that $\psi \cdot z$ is $Z(\hat{G})$-equivalent to $\psi$ if and only if $\alpha$ belongs to the sum of the following two subgroups $\operatorname{of}^{1}(F, Z(\hat{H}))$ :

$$
\begin{gathered}
\operatorname{im}\left[\operatorname{ker}^{1}(F, Z(\hat{G})) \rightarrow \operatorname{ker}^{1}(F, Z(\hat{H}))\right], \\
\operatorname{im}\left[\widetilde{S}_{\psi} \rightarrow \operatorname{ker}^{1}(F, Z(\hat{H}))\right]
\end{gathered}
$$

(see 10.2 for the definition of $\Im_{\psi} \rightarrow \operatorname{ker}^{1}(F, Z(\hat{H}))$ ). We need some temporary notation. We will write $A$ for $\operatorname{cok}\left[\operatorname{ker}^{1}(F, Z(\hat{G})) \rightarrow \operatorname{ker}^{1}(F, Z(\hat{H}))\right]$ and $B$ for the cokernel of the composition

$$
\mathfrak{S}_{\psi} \rightarrow \operatorname{ker}^{1}(F, Z(\hat{H})) \rightarrow A,
$$

where $\operatorname{ker}^{1}(F, Z(\hat{H})) \rightarrow A$ is the canonical map. From the previous discussion we see that the number of $Z(\hat{G})$-equivalence classes contained in $[\psi]$ is equal to $|B|$.

The definition of $B$ gives us an exact sequence

$$
\Im_{\psi} \rightarrow A \rightarrow B \rightarrow 1 \text {. }
$$

Consider the homomorphism ${ }_{G} \Im_{\psi} \rightarrow \Im_{\psi}$ induced by the inclusion ${ }_{G} S_{\psi} \subset S_{\psi}$. An easy calculation shows that

$$
\operatorname{im}\left[{ }_{G} \Im_{\psi} \rightarrow \mathbb{S}_{\psi}\right]=\operatorname{ker}\left[\mathfrak{S}_{\psi} \rightarrow A\right]
$$

Another easy calculation, which uses the fact that $(H, s, \eta)$ and $\psi$ are elliptic, shows that

$$
\operatorname{ker}\left[{ }_{G} \mathfrak{\Im}_{\psi} \rightarrow \Im_{\psi}\right]=\Re(H / F)
$$


(see 7.4 for the definition of $\Re(H / F)$ ). Putting together (11.2.3.3), (11.2.3.4), (11.2.3.5), we get an exact sequence

$$
1 \rightarrow \Re(H / F) \rightarrow{ }_{G} \mathfrak{S}_{\psi} \rightarrow \mathfrak{S}_{\psi} \rightarrow A \rightarrow B \rightarrow 1,
$$

which implies

$$
|B|=\left.\left.|A| \cdot|\Re(H / F)|^{-1} \cdot\right|_{G} \Im_{\psi}|\cdot| \Im_{\psi}\right|^{-1} .
$$

Consider the exact sequence

$$
1 \rightarrow Z(\hat{G}) \rightarrow Z(\hat{H}) \rightarrow Z(\hat{H}) / Z(\hat{G}) \rightarrow 1,
$$

and apply Corollary 2.3. As in the proof of Lemma 8.3.2 we get an exact sequence

$$
\begin{aligned}
1 & \rightarrow \pi_{0}\left(Z(\hat{G})^{\Gamma}\right) \rightarrow \pi_{0}\left(Z(\hat{H})^{\Gamma}\right) \rightarrow \Re(H / F) \\
& \rightarrow \operatorname{ker}^{1}(F, Z(\hat{G})) \rightarrow \operatorname{ker}^{1}(F, Z(\hat{H})) \rightarrow A \rightarrow 1 .
\end{aligned}
$$

Note that the injectivity of

$$
\pi_{0}\left(Z(\hat{G})^{\Gamma}\right) \rightarrow \pi_{0}\left(Z(\hat{H})^{\Gamma}\right)
$$

is a consequence of our assumption that $(H, s, \eta)$ is elliptic. The formula (5.1.1) for relative Tamagawa numbers, together with the exact sequence above, implies

$$
|A| \cdot|\Re(H / F)|^{-1}=\tau_{1}(G) \cdot \tau_{1}(H)^{-1} .
$$

Combining (11.2.3.7) and (11.2.3.8) finishes the proof.

11.3. Assume that $G_{\mathrm{der}}$ is simply connected. Choose a set $\mathscr{E}$ of representatives for the isomorphism classes of elliptic endoscopic triples for $G$, and for each $(H, s, \eta) \in \mathscr{E}$ choose an $L$-homomorphism $\eta^{\prime}:{ }^{L} H \rightarrow{ }^{L} G$ that extends $\eta: \hat{H} \rightarrow \hat{G}$. Our assumption that $G_{\text {der }}$ is simply connected guarantees that such extensions $\eta^{\prime}$ exist (see [L1]). Let $\psi: W_{F} \rightarrow^{L} H$ be an admissible homomorphism, and let $\varphi=\eta^{\prime} \circ \psi$. Then $\eta(s) \in S_{\varphi}$, Write $\epsilon$ for the image of $\eta(s)$ in $\widetilde{S}_{\varphi}$ and $[\epsilon]$ for the conjugacy class of $\epsilon$ in $\mathfrak{S}_{\varphi}$. Then the map $\psi \mapsto(\varphi, \epsilon)$ induces a map

$$
[[\psi]] \mapsto([\varphi],[\epsilon])
$$

from the set of $Z(\hat{G})$-equivalence classes $[[\psi]]$ of admissible homomorphisms for $H$ to the set of pairs $([\varphi],[\epsilon])$, where $[\varphi]$ is an equivalence class of admissible homomorphisms for $G$ and $[\epsilon]$ is a conjugacy class in $\mathfrak{S}_{\varphi}$ (note that if $\varphi^{\prime}$ also belongs to $[\varphi]$, then there is an isomorphism $\widetilde{S}_{\varphi} \stackrel{\rightarrow}{\rightarrow} \widetilde{S}_{\varphi^{\prime}}$, canonical up to inner automorphisms, and therefore the set of conjugacy classes in $\widetilde{S}_{\varphi^{\prime}}$ is independent of $\left.\varphi^{\prime} \in[\varphi]\right)$. 
11.3.2. Proposition. Let $\varphi$ be an elliptic admissible homomorphism for $G$, and let $\epsilon$ be an element of $\mathfrak{S}_{\varphi}$. Write $\left(\mathfrak{S}_{\varphi}\right)_{\epsilon}$ for the centralizer of $\epsilon$ in $\mathfrak{S}_{\varphi}$. Then

$$
\left|\left(\Im_{\varphi}\right)_{\epsilon}\right|^{-1}=\left.\left.\sum \sum \lambda(H, s, \eta)^{-1} \cdot\right|_{G} \Im_{\psi}\right|^{-1},
$$

where the first sum is taken over $(H, s, \eta) \in \mathscr{E}$ and the second sum is taken over a set of representatives $\psi$ for the $Z(\hat{G})$-equivalence classes of admissible homomorphisms for $H$ that map to $([\varphi],[\epsilon])$ under (11.3.1).

We will use the proposition when we discuss the stabilization of the trace formula. The remainder of this section will be devoted to a proof of the proposition.

11.3.3. Let $A$ denote the group of continuous 1-cocycles of $W_{F}$ in $Z(\hat{G})$ whose class in $H^{1}\left(W_{F}, Z(\hat{G})\right)$ is locally trivial. The group $A$ acts on the set of admissible homomorphisms $\psi$ for $H$. Let $\langle\psi\rangle$ denote the orbit of $\psi$ under $A$. The group $A$ also acts on the set of admissible homomorphisms $\varphi$ for $G$. Let $\langle\varphi\rangle$ denote the orbit of $\varphi$ under $A$.

Let $U$ denote the set of quadruples $\left(s, \rho_{0}, \xi,\langle\psi\rangle\right)$ satisfying the following conditions:

(a) $s$ is a semisimple element of $\hat{G} / Z(\hat{G})$, and $\rho_{0}$ is an $L$-action of $\Gamma$ on $\hat{G}_{s}^{0}$ such that $(s, \rho)$ is an elliptic endoscopic datum for $G$, where $\rho$ is the composition $\Gamma \stackrel{\rho_{0}}{\rightarrow} \operatorname{Aut}\left(\hat{G}_{s}^{0}\right) \rightarrow \operatorname{Out}\left(\hat{G}_{s}^{0}\right)$.

(b) $\xi:{ }^{L} H \rightarrow{ }^{L} G$ is an $L$-homomorphism that extends the inclusion $\hat{H} \rightarrow \hat{G}$, where $\hat{H}$ is $\hat{G}_{s}^{0}$ and ${ }^{L} H$ is the semi-direct product of $W_{F}$ and $\hat{H}$ provided by $\rho_{0}$.

(c) $\psi: W_{F} \rightarrow{ }^{L} H$ is an admissible homomorphism such that $\xi \circ \psi$ is elliptic for $G$.

Let $V$ denote the set of triples $\left(s, \rho_{0}, \xi\right)$ satisfying (a) and (b). There is an obvious map $p: U \rightarrow V$.

Let $Y$ denote the set of elliptic endoscopic data $(s, \rho)$. There is an obvious map $g: V \rightarrow Y$.

Let $X$ denote the set of pairs $(\langle\varphi\rangle, \xi)$, where $\varphi$ is an elliptic admissible homomorphism for $G$ and $\epsilon \in \mathbb{S}_{\varphi}$ (note that $\mathbb{S}_{\varphi}$ is independent of the representative $\varphi$ of $\langle\varphi\rangle$ ).

We define a map $f: U \rightarrow X$ by sending $\left(s, \rho_{0}, \xi,\langle\psi\rangle\right)$ to $(\langle\xi \circ \psi\rangle, s)$, and we define a map $q: X \rightarrow Y$ by sending $(\langle\varphi\rangle, \epsilon)$ to $(\epsilon, \bar{\varphi})$, where $\bar{\varphi}$ is the unique homomorphism $\bar{\varphi}: \Gamma \rightarrow \operatorname{Out}\left(\hat{G}_{\epsilon}^{0}\right)$ such that the diagram

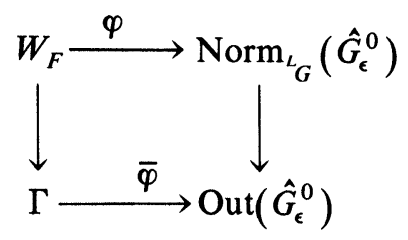

commutes.

The group $\hat{G} / Z(\hat{G})$ acts on $U, V, X, Y$. The four maps we have defined are all 
$\hat{G} / Z(\hat{G})$-maps and give us a commutative diagram

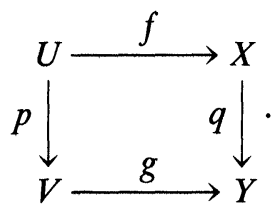

It is not hard to check that this square is Cartesian (i.e., it makes $U$ into the product of $V$ and $X$ over $Y$ ). The map $g$ is surjective, since we are assuming that $G_{\text {der }}$ is simply connected. Proposition 11.3.2 now follows from Lemma 11.4.1 below.

11.4. Let $G$ be an abstract group. Consider a Cartesian square (11.3.3.1) of $G$-sets. Assume that $g$ is surjective and that the stabilizer $G_{x}$ of $x$ in $G$ is finite for all $x \in X$. Suppose that we are given, for each $y \in Y$, a subgroup $H(y)$ of finite index in $G_{y}$. For $v \in V$ we write $H(v)$ for $H(g(v))$ and use the bijection $p^{-1}(v) \stackrel{\sim}{\rightarrow} q^{-1}(g(v))$ induced by $f$ to transport the action of $H(v)$ on $q^{-1}(g(v))$ over to $p^{-1}(v)$.

11.4.1. Lemma. For any $x \in X$ and any cross section $V_{0} \subset V$ of the composition $V \rightarrow Y \rightarrow G \backslash Y$ we have

$$
\left|G_{x}\right|^{-1}=\sum_{v \in V_{0}} \sum_{u \in U_{x, v}}\left[G_{g(v)}: H(v)\right]^{-1} \cdot\left|H(v)_{u}\right|^{-1}
$$

where $U_{x, v}=H(v) \backslash\left\{u \in p^{-1}(v): f(u) \in G \cdot x\right\}$.

We reduce immediately to the case in which $V=Y, U=X, f=\mathrm{id}_{X}, g=\mathrm{id}_{Y}$, and $G$ acts transitively on $X$ and $Y$. Then $V_{0}$ has only one element, and without loss of generality we may assume that $V_{0}=\{y\}$, where $y=q(x)$. Then the formula of the lemma is just a reflection of the decomposition

$$
H(y) \backslash G_{y}=\coprod_{g} H(y) \backslash H(y) g G_{x},
$$

where $g$ runs over a set of representatives for $H(y) \backslash G_{y} / G_{x}$.

11.4.2. To apply the lemma to the proof of the proposition, we need to choose subgroups $H(y)$ of finite index in $(\hat{G} / Z(\hat{G}))_{y}$ for $y=(s, \rho) \in Y$. But $(\hat{G} / Z(\hat{G}))_{y}=\operatorname{Aut}(s, \rho) / Z(\hat{G})$, since $(s, \rho)$ is elliptic (see 7.3), and we take $H(y)$ to be $\hat{H} / Z(\hat{G})$. Let $x=(\langle\varphi\rangle, \epsilon) \in X$. The $(\hat{G} / Z(\hat{G}))_{x}=\left(\Im_{\varphi}\right)_{\epsilon}$, since $\varphi$ is elliptic. Let $v=\left(s, \rho_{0}, \xi\right) \in V$. Then $p^{-1}(v)$ can be identified with the set of $\langle\psi\rangle$ such that $\psi$ is an admissible homomorphism $W_{F} \rightarrow^{L} H$ and $\xi \circ \psi$ is elliptic. The action of $H(v)=\hat{H} / Z(\hat{G})$ on $p^{-1}(v)$ is the obvious conjugation action. Let $u=\left(s, \rho_{0}, \xi\right.$, $\langle\psi\rangle) \in p^{-1}(v)$. Then $H(v)_{u}={ }_{G} \Im_{\psi}$, since the fact that $\xi \circ \psi$ is elliptic implies that ${ }_{G} S_{\psi}^{0} \subset Z(\hat{G})$. We leave the verification of the remaining details to the reader.

12. Stable trace formula. This section is purely speculative. We will try to understand the stabilization of the contribution to the trace formula made by 
automorphic representations corresponding to elliptic tempered admissible homomorphisms. It is impossible for us to prove anything without making a number of assumptions, whose correctness seems impossible to verify at the present time. Nevertheless, the theory of automorphic representations is difficult enough that it is not without interest to guess how some of its parts fit together. In our discussion we will assume that the reader is familiar with Langlands's work on the stable trace formula [L3].

Let $F$ be a number field, and let $G$ be a connected reductive group over $F$. Assume that $G_{\text {der }}$ is simply connected, so that $\hat{H} \rightarrow \hat{G}$ can be extended to ${ }^{L} H \rightarrow{ }^{L} G$ for every endoscopic group $H$ for $G$ [L1]. Let ${ }_{0} Z$ be a closed subgroup of $Z(A)$, where $Z=Z(G)$, such that ${ }_{0} Z \cdot Z(F)$ is closed and ${ }_{0} Z \cdot Z(F) \backslash Z(\mathrm{~A})$ is compact. Assume that ${ }_{0} Z=\prod_{v 0} Z_{v}$. Let $\chi$ be a character of ${ }_{0} Z$, trivial on ${ }_{0} Z \cap Z(F)$. We get $L_{\chi}^{2}(G(F) \backslash G(\mathrm{~A}))$, and we have a trace formula for suitable functions $f=\prod_{v} f_{v}$ on $G(\mathrm{~A})$.

Let $\mathscr{E}$ be a set of representatives for the isomorphism classes of elliptic endoscopic triples for $G$, and for each $(H, s, \eta) \in \mathscr{E}$ choose an $L$-homomorphism $\eta^{\prime}:{ }^{L} H \rightarrow{ }^{L} G$ that extends $\eta: \hat{H} \rightarrow \hat{G}$.

Under the assumption that there exists, for each $(H, s, \eta) \in \mathscr{E}$, a function $f^{H}$ on $H(\mathrm{~A})$ with suitable orbital integrals, Langlands [L3] proves a formula

$$
T_{e}(f)=\sum_{(H, s, \eta) \in \mathscr{E}} \iota(G, H) S T_{e}\left(f^{H}\right) .
$$

Here $T_{e}(f)$ is the elliptic regular part of the trace formula for $f$, and $S T_{e}\left(f^{H}\right)$ is the elliptic $G$-regular part of the stable trace formula for $f^{H}$.

We would like to have the same result for the other side of the trace formula. That is, we want to have

$$
T_{c}(f)=\sum_{(H, s, \eta) \in \mathscr{E}} \iota(G, H) S T_{c}\left(f^{H}\right)
$$

where $T_{c}(f)$ is the tempered cuspidal part of the trace formula for $f$ and $S T_{c}\left(f^{H}\right)$ is the tempered $G$-cuspidal part of the trace formula for $f^{H}$. In order to account for all of the tempered cuspidal automorphic representations of $G$, Langlands [L2] has suggested the existence of a group even larger than the Weil group $W_{F}$ of $F$. We will consider a variant $L_{F}$ of this conjectural group, and we suggest that this group be named the Langlands group. The group $L_{F}$ should be an extension of $W_{F}$ by a compact group. It should satisfy axioms $\left(W_{1}\right),\left(W_{2}\right),\left(W_{3}\right)$ of [T3] (but not $\left(W_{4}\right)$, of course). For the local fields $F_{v}$ it is known that $L_{F_{v}}$ should be:

(a) $L_{F_{\mathrm{v}}}=W_{F_{\mathrm{v}}}$ if $v$ is archimedean,

(b) $L_{F_{\mathrm{v}}}=W_{F_{\mathrm{v}}} \times \mathrm{SU}(2, \mathrm{R})$ if $v$ is nonarchimedean.

There should be a natural bijection between the irreducible $n$-dimensional complex representations of $L_{F}$ (resp. $L_{F_{\mathrm{v}}}$ ) and the cuspidal automorphic representations of $\mathrm{GL}_{n}(\mathrm{~A})$ (resp. irreducible admissible representations of $\left.\mathrm{GL}_{n}\left(F_{v}\right)\right)$. There should be homomorphisms $L_{F_{\mathrm{r}}} \rightarrow L_{F}$, canonical up to 
conjugation by elements of $L_{F}$. The definitions and results of Sections 10 and 11 can be extended to $L_{F}$ (if one grants the properties above), since the properties of $W_{F}$ that we used are shared by $L_{F}$.

Following Labesse-Langlands [L-L], we will guess that the tempered cuspidal part of $L_{\chi}^{2}(G(F) \backslash G(\mathrm{~A}))$ is isomorphic to

$$
\bigoplus_{[\varphi]} \bigoplus_{\pi \in \Pi(\varphi)} m(\varphi, \pi) \cdot \pi
$$

where $m(\varphi, \pi)=\left|\Im_{\varphi}\right|^{-1} \sum_{\epsilon \in \mathfrak{S}_{\varphi}}\langle\epsilon, \pi\rangle$. This expression requires some explanation. The symbol $[\varphi]$ stands for the equivalence class (10.4) of an admissible homomorphism $\varphi: L_{F} \rightarrow^{L} G$, and the first direct sum is taken over all equivalence classes of elliptic tempered $\varphi$ such that the associated character $\chi_{\varphi}$ of $Z(A)$ agrees with $\chi$ on ${ }_{0} Z$. Note that $\chi_{\varphi}$ depends only on $[\varphi]$. The symbol $\Pi(\varphi)$ stands for the $L$-packet of $\varphi$. These $L$-packets should be defined so that $\pi=\bigotimes_{v} \pi_{v}$ belongs to $\Pi(\varphi)$ if and only if $\pi_{v} \in \Pi\left(\varphi_{v}\right)$ for all $v$ and $\pi_{v}$ is $K_{v}$-spherical for almost all $v$ $\left(K=\Pi_{v} K_{v}\right.$ is some maximal compact subgroup of $\left.G(\mathrm{~A})\right)$. We should have

$$
\langle\epsilon, \pi\rangle=\prod_{v}\left\langle\epsilon_{v}, \pi_{v}\right\rangle
$$

where $\epsilon_{v}$ is the image of $\epsilon$ under $\mathfrak{S}_{\varphi} \rightarrow \mathfrak{S}_{\varphi_{v}}$. The local functions $<$, \rangle$: \Im_{\varphi_{v}} \times \Pi\left(\varphi_{v}\right) \rightarrow \mathrm{C}$ are somewhat noncanonical, but the global function $\langle$,$\rangle is$ canonical. One expects that the complex-valued functions $\epsilon_{v} \mapsto\left\langle\epsilon_{v}, \pi_{v}\right\rangle$ on $\widetilde{S}_{\varphi_{v}}$ are characters of nonzero (possibly reducible) finite dimensional complex representations of $\mathfrak{S}_{\varphi_{v}}$. We will need to normalize the local functions $\langle$,$\rangle so$ that for almost all $v$ we have $\left\langle\epsilon_{v}, \pi_{v}\right\rangle=1$ whenever $\pi_{v}$ is $K_{v}$-spherical. Assuming all of this, we see that $m(\pi, \varphi)$ is a nonnegative integer.

Using (12.3), we get the following formula:

$$
T_{c}(f)=\sum_{[\varphi]}\left|S_{\varphi}\right|^{-1} \sum_{\pi \in \Pi(\varphi)} \sum_{\epsilon \in \mathbb{S}_{\varphi}}\langle\epsilon, \pi\rangle \operatorname{tr} \pi(f) .
$$

We also assume that

$$
S T_{c}(f)=\sum_{[\varphi]}\left|\Im_{\varphi}\right|^{-1} \sum_{\pi \in \Pi(\varphi)}\langle 1, \pi\rangle \operatorname{tr} \pi(f) .
$$

Let $(H, s, \eta) \in \mathscr{E}$. Recall that we have chosen an extension $\eta^{\prime}:{ }^{L} H \rightarrow{ }^{L} G$ of $\eta$. Let $\psi: L_{F} \rightarrow{ }^{L} H$ be a tempered admissible homomorphism, and let $\varphi=\eta^{\prime} \circ \psi$. Then the function $f^{H}$ should have the property that

$$
\sum_{\pi \in \Pi(\psi)}\langle 1, \pi\rangle \operatorname{tr} \pi\left(f^{H}\right)=\sum_{\pi \in \Pi(\varphi)}\left\langle\epsilon_{s}, \pi\right\rangle \operatorname{tr} \pi(f),
$$

where $\epsilon_{s}$ is the image of $\eta(s) \in S_{\varphi}$ in $\widetilde{S}_{\varphi}$. Thus the stabilization (12.2) that we are 
trying to obtain is equivalent to the equality of

$$
\sum_{(H, s, \eta) \in \delta} \iota(G, H) \sum_{[\psi]}\left|\Im_{\psi}\right|^{-1} \sum_{\pi \in \Pi\left(\eta^{\prime} \circ \psi\right)}\left\langle\epsilon_{s}, \pi\right\rangle \operatorname{tr} \pi(f)
$$

and

$$
\sum_{(\varphi)}\left|\Im_{\varphi}\right|^{-1} \sum_{\pi \in \Pi(\varphi)} \sum_{\epsilon \in \mathbb{S}_{\varphi}}\langle\epsilon, \pi\rangle \operatorname{tr} \pi(f)
$$

The class $\left[\eta^{\prime} \circ \psi\right]$ is not determined by the class $[\psi]$. This is why we introduced $Z(\hat{G})$-equivalence $(11.1)$. We write $[[\psi]]$ for the $Z(\hat{G})$-equivalence class of $\psi$. Whether or not $\eta^{\prime} \circ \psi$ is elliptic should only depend on $[\psi]$. In (12.8) we sum only over $[\psi]$ such that $\eta^{\prime} \circ \psi$ is elliptic. Using Proposition 11.2.1 and Theorem 8.3.1, we can rewrite $(12.8)$ as

$$
\left.\left.\sum_{(H, s, \eta) \in \mathscr{E}} \lambda(H, s, \eta)^{-1} \sum_{[[\psi]]}\right|_{G} \Im_{\psi}\right|^{-1} \sum_{\pi \in \Pi\left(\eta^{\prime} \circ \psi\right)}\left\langle\epsilon_{s}, \pi\right\rangle \operatorname{tr} \pi(f) .
$$

Let $\varphi$ be an admissible homomorphism $L_{F} \rightarrow^{L} G$ such that [ $\left.\varphi\right]$ appears in (12.9), and let $\epsilon \in \mathbb{S}_{\varphi}$. Let $[\epsilon]$ denote the conjugacy class of $\epsilon$ in $\Im_{\varphi}$, and let $\left(\Im_{\varphi}\right)_{\epsilon}$ denote the centralizer of $\epsilon$ in $\mathfrak{S}_{\varphi}$. The contribution of $[\varphi],[\epsilon]$ to $(12.9)$ is the product of

$$
\sum_{\pi \in \Pi(\varphi)}\langle\epsilon, \pi\rangle \operatorname{tr} \pi(f)
$$

and

$$
\left|\left(\Im_{\varphi}\right)_{\epsilon}\right|^{-1}
$$

The contribution of $[\varphi],[\epsilon]$ to $(12.10)$ is the product of

$$
\sum_{\pi \in \Pi(\varphi)}\langle\epsilon, \pi\rangle \operatorname{tr} \pi(f)
$$

and

$$
\left.\left.\sum_{(H, s, \eta) \in \mathscr{\delta}} \lambda(H, s, \eta)^{-1} \sum_{[[\psi]]}\right|_{G} \Im_{\psi}\right|^{-1}
$$

where the last sum is taken over those $[[\psi]]$ that map to $([\varphi],[\epsilon])$ under (11.3.1). However, Proposition 11.3.2 tells us that the (12.11) and (12.12) are equal. Therefore (12.9) and (12.10) are equal. This finishes the stabilization of the tempered cuspidal part of the trace formula for $G$.

\section{REFERENCES}

[B] A. Borel, Automorphic L-functions, Automorphic Forms, Representations and L-functions, Proc. Sympos. Pure Math., vol. 33, part 2, Amer. Math. Soc., Providence, R.I., 1979, pp. 27-61. 
[C-E] H. Cartan and S. Eilenberg, Homological Algebra, Princeton Univ. Press., Princeton, N.J., 1956.

[H] G. Hochschild, The Structure of Lie Groups, Holden-Day, Inc., San Francisco, 1965.

[Ho] R. Hoobler, A cohomological interpretation of Brauer groups of rings, Pacific J. Math. 86 (1980), 89-92.

[K] R. KotTwITz, Rational conjugacy classes in reductive groups, Duke Math. J. 49 (1982), 785-806.

[Kn] M. KNESER, Galoiskohomologie halbeinfacher algebraischer Gruppen über p-adischen Körpern, I., Math. Zeit. 88 (1965), 40-47; II, Math. Zeit. 89 (1965), 250-272.

[L-L] J-P. LabesSe and R. P. Langlands, L-indistinguishability for SL(2), Can. J. Math. 31 (1979), 726-785.

[L1] R. P. Langlands, Stable conjugacy: definitions and lemmas, Can. J. Math. 31 (1979), 700-725.

[L2] - Automorphic representations, Shimura varieties, and motives, Automorphic Forms, Representations and $L$-functions, Proc. Sympos. Pure Math., vol. 33, part 2, Amer. Math. Soc., Providence, R.I., 1979, pp. 205-246.

[L3] - Les débuts d'une formule des traces stable, Publ. Math. de l'Univ. Paris VII, vol. 13, Paris, 1983.

[M-S] J. S. Milne AND K.-Y. SHIH, “Conjugates of Shimura varieties," in Hodge Cycles, Motives and Shimura Varieties, Lecture notes in mathematics 900, Springer-Verlag, 1982, pp. 280-356.

[O] T. Ono, On Tamagawa numbers, Algebraic Groups and Discontinuous Subgroups, Proc. Sympos. Pure Math., vol. 9, Amer. Math. Soc., Providence, R.I., 1966, pp. 122-132.

[R] M. Rosenlicht, Toroidal algebraic groups, Proc. Amer. Math. Soc. 12 (1961), 984-988.

[Sa] J.-J. SANSUC, Groupe de Brauer et arithmétique des groupes algébriques linéaires sur un corps de nombres, J. Reine Angew. Math. 327 (1981), 12-80.

[S] S. S. Shatz, Profinite Groups, Arithmetic and Geometry, Annals of Math. Studies 67, Princeton, 1972.

[Sh] D. ShElSTAD, L-indistinguishability for real groups, Math. Ann. 259 (1982), 385-430.

[T1] J. TATE, Duality theorems in Galois cohomology over number fields, Proc. International Congress Math., Stockholm (1962), 288-295.

[T2] The cohomology groups of tori in finite Galois extensions of number fields, Nagoya Math. J. 27 (1966), 709-719.

[T3] - Number theoretic background, Automorphic Forms, Representations and $L$ functions, Proc. Sympos. Pure Math., vol. 33, part 2, Amer. Math. Soc., Providence, R.I., 1979, pp. 3-26.

[Ti] J. TITs, Reductive groups over local fields, Automorphic Forms, Representations and $L$-functions, Proc. Sympos. Pure. Math., vol. 33, part 1, Amer. Math. Soc., Providence, R.I., 1979, pp. 29-69.

Department of Mathematics, University of Washington, Seattle, Washington 98195 